\title{
Optimal Decisions for Prepositioning Emergency Supplies Problem with Type-2 Fuzzy Variables
}

\author{
Xuejie Bai ${ }^{1,2}$ \\ ${ }^{1}$ College of Science, Hebei Agricultural University, Baoding, Hebei 071001, China \\ ${ }^{2}$ College of Management, Hebei University, Baoding, Hebei 071002, China \\ Correspondence should be addressed to Xuejie Bai; hebaubxj@163.com
}

Received 4 June 2016; Accepted 14 September 2016

Academic Editor: Lu Zhen

Copyright (c) 2016 Xuejie Bai. This is an open access article distributed under the Creative Commons Attribution License, which permits unrestricted use, distribution, and reproduction in any medium, provided the original work is properly cited.

\begin{abstract}
Prepositioning emergency supplies serves an important function in disaster relief operations. This paper presents a new class of fuzzy prepositioning emergency supplies model for three-echelon humanitarian logistics network, in which the postdisaster acquisition and transportation costs, the suppliers' supply, and affected areas' demand are uncertain and characterized by type2 fuzzy variables with known possibility distributions. Since the inherent complexity of fuzzy prepositioning problem may be troublesome, the existing methods are no longer effective in dealing with the proposed model directly. We first derive the optimistic and pessimistic values formula for credibility value-at-risk (CVaR) reduced fuzzy variable of type-2 trapezoidal fuzzy variable. On the basis of formula obtained, we can convert original fuzzy prepositioning model into its equivalent parametric mixed integer programming form, which can be solved by conventional algorithms or general-purpose software. Finally, some numerical experiments have been performed to illustrate the effectiveness of the proposed model and solution strategy.
\end{abstract}

\section{Introduction}

Disasters caused massive casualties and tremendous economic and social damage. Much infrastructure lacking adequate structural stability collapses and human beings either die or need immediate help. These huge losses make emergency logistics management attract considerable research attention from both logistics academics and practitioners in recent years. More and more optimization models and algorithms are powerful tools to handle this kind of problems. Abounacer et al. [1] presented a three-objective locationtransportation model for disaster response and adopted an epsilon-constraint method for generating the exact Pareto frontier. Barzinpour and Esmaeili [2] developed a multiobjective relief chain location distribution model for preparation planning phase of disaster management which was applied to a real case study of an urban district in Iran. Extensive research has been documented on emergency logistics and disaster operations management. For more details, please refer to Altay and Green [3], Galindo and
Batta [4], Anaya-Arenas et al. [5], and Özdamar and Ertem [6].

When creating an efficient and effective emergency plan, uncertainty is a paramount challenge. As pointed out by Bozorgi-Amiri et al. [7], information about demand, supply, and cost is unknown in advance due to the lack of related data. The existence of uncertainty has motivated some researchers to address the critical parameters in the form of stochastic approaches. For example, Salmerón and Apte [8] developed a two-stage stochastic optimization model for natural disaster asset prepositioning problem under the uncertainty of the event's location and severity. Rawls and Turnquist [9] formulated a two-stage stochastic mixed integer programming model that determined the facility location and amounts of various emergency supplies to be prepositioned and designed a heuristic solution approach called the Lagrangian L-shaped method to solve this problem. Balcik and Ak [10] studied the supplier selection problem in preparation for the sudden-onset disasters and developed a stochastic programming model by using a scenario-based method to 
represent demand uncertainty. In order to study the emergency medical services design problem under uncertainty, Zhang and Jiang [11] employed a robust counterpart approach and found model's Pareto-optimal solutions for costs and response times by the weighting method. Caunhye et al. [12] proposed a two-stage stochastic location-routing model that integrated preparedness and response schedule under uncertainties in demand and the state of the infrastructure. For a thorough coverage of stochastic disaster management problem, the interested reader may refer to Hoyos et al. [13] and references therein.

Fuzzy optimization techniques have been developed for emergency supplies management problem to model uncertainties mathematically. For example, Sheu [14] employed a hybrid fuzzy clustering optimization approach to a threelayer emergency logistics codistribution conceptual framework and demonstrated the applicability of the new method and potential advantages through numerical studies. Sun et al. [15] used the fuzzy set and rough set theory to build an emergency material demand prediction model and developed decision rules and computing methods based on the risk decision-making principle of classical operational research. Considering the multiple attributes of disaster relief supply chains, Zheng and Ling [16] proposed a multiobjective fuzzy emergency transportation planning model and designed a cooperative optimization method to solve efficiently the proposed problem. Ruan et al. [17] proposed a method for comparing triangular fuzzy numbers by combining a preference-based index and $\alpha$-cut idea and developed an approach for proportionately allocating limited relief supplies to emergency distribution points. Bai [18] proposed a two-stage emergency supplies allocation model to examine the impact of uncertain demands of the affected areas and the availability of path and supply amounts on the optimal allocation plan. Tofighi et al. [19] established two-stage possibilistic-stochastic programming that determined the locations of central warehouses and local distribution centers and designed a tailored differential evolution algorithm to find good enough feasible solution.

In summary, the existing studies with regard to emergency supplies mainly depended on the deterministic optimization method. Although there were some references that employed the stochastic approaches or fuzzy techniques to deal with the uncertainty, they characterized the uncertain information with fixed probability or possibility distributions. In the case that the fixed distributions cannot be determined exactly in the real problem, then it is unsuitable to adopt the obtained solution to conduct the emergency management. In this paper, we propose a new parametric method to express uncertain parameters in the prepositioning emergency supplies problem by using variable possibility distributions instead of fixed possibility distributions. The variable possibility distributions are given by credibility value-at-risk (VaR) reduction method to the type-2 fuzzy variables. The type- 2 fuzzy variable was introduced by Liu and Liu [20] as an extension of the type-2 fuzzy set [21] to depict the fuzzy phenomenon. Furthermore, four kinds of major reduction methods were refined for type- 2 fuzzy variables to reduce the uncertainty embedded in the secondary possibility distributions. The mean value reduction method was given by Choquet fuzzy integrals of regular fuzzy variables [22]. The critical value reduction method was defined by Sugeno fuzzy integrals of regular fuzzy variables [23]. The equivalent value reduction method was introduced via classic Lebesgue-Stieltjes integrals of regular fuzzy variables [24] and the VaR reduction method was based on the possibility VaR or credibility VaR of regular fuzzy variables $[25,26]$. For the recent development and other applications of type-2 fuzzy theory, we refer the reader to [27, 28] for detailed discussion. The theoretical development above motivates us to study prepositioning emergency supplies problem from a new perspective. Bai [29] addressed a fuzzy prepositioning problem for emergency supplies on the basis of fuzzy possibility theory, where the transportation costs, the suppliers' supply amounts, the affected areas' demands, and the capacities of the roads were characterized by type2 triangular fuzzy variables. This article differs from the mentioned work in exploring the decision maker's risk-averse attitude towards uncertainty in place of the risk-neutral criterion.

This paper aims to adopt fuzzy parametric optimization method to address the prepositioning emergency supplies problem. To the best of our knowledge, this kind of prepositioning problem with variable possibility distribution has not yet been studied in the existing literature. The main contributions of the paper are summarized as follows: (1) The postdisaster acquisition and transportation costs, the suppliers' supply, and affected areas' demand are unknown prior to the event and are characterized by type- 2 fuzzy variables. This paper applies fuzzy optimization to examine the impact of the uncertain parameters on the optimal allocation plan. (2) This paper builds a fuzzy prepositioning emergency supplies model with risk-averse objective function and credibility constraints in a framework of type- 2 fuzzy theory. (3) Our fuzzy prepositioning emergency supplies model itself is difficult to solve because it has a maximal credibility objective and several credibility constraints. We use the optimistic and pessimistic value formula to obtain an equivalent deterministic form without sacrificing optimality. This allows the original model to be turned to a tractable one computationally that can be solved by generic software. (4) We conduct numerical example in the context of emergency logistics to show the value of fuzzy model and the efficiency of the presented method.

The rest of this paper is organized as follows. Section 2 derives the pessimistic and optimistic value formula of the reduced fuzzy variables for common type- 2 trapezoidal fuzzy variable. Section 3 formulates a new class of fuzzy prepositioning emergency supplies problem with maximal credibility criterion. In Section 4, making use of the results obtained, we convert the original prepositioning problem to its equivalent model. In Section 5, the proposed approach is applied to a practical fuzzy prepositioning example, and the computational results show the superiority of parametric method. Finally, Section 6 gives the conclusions. 


\section{Optimistic and Pessimistic Values of CVaR Reduced Variable}

Let $(\Gamma, \mathscr{A}, \widetilde{P}$ os) be a fuzzy possibility space and $\widetilde{\xi}$ a type-2 fuzzy variable with secondary possibility distribution $\widetilde{\mu}_{\tilde{\xi}}(x)$. To reduce the uncertainty in $\widetilde{\mu}_{\tilde{\xi}}(x)$, we employ the CVaR of $\widetilde{P} o s\{\tilde{\xi}=x\}$ as the representing value of $\widetilde{\mu}_{\tilde{\xi}}(x)$. The method is referred to as the CVaR reduction [26]. The reduced fuzzy variable obtained by $\mathrm{CVaR}$ reduction method is denoted by $\xi$.

Let $\tilde{\xi}=\left(\widetilde{r}_{1}, \widetilde{r}_{2}, \widetilde{r}_{3}, \widetilde{r}_{4} ; \theta_{l}, \theta_{r}\right)$ be a type-2 trapezoidal fuzzy variable, and $\theta=\left(\theta_{l}, \theta_{r}\right)$. If $\alpha \in(0,0.5]$, then the CVaR

$$
\mu_{\xi}(x ; \theta, \alpha)= \begin{cases}\left(1-\theta_{r}+2 \alpha \theta_{r}\right) \frac{x-r_{1}}{r_{2}-r_{1}}, & \text { if } x \in\left[r_{1}, \frac{r_{1}+r_{2}}{2}\right] \\ \frac{\left(1+\theta_{r}-2 \alpha \theta_{r}\right) x-(1-2 \alpha) \theta_{r} r_{2}-r_{1}}{r_{2}-r_{1}}, & \text { if } x \in\left[\frac{r_{1}+r_{2}}{2}, r_{2}\right] \\ 1, & \text { if } x \in\left[r_{2}, r_{3}\right] \\ \frac{-\left(1+\theta_{r}-2 \alpha \theta_{r}\right) x+(1-2 \alpha) \theta_{r} r_{3}+r_{4}}{r_{4}-r_{3}}, & \text { if } x \in\left[r_{3}, \frac{r_{3}+r_{4}}{2}\right] \\ \left(1-\theta_{r}+2 \alpha \theta_{r}\right) \frac{r_{4}-x}{r_{4}-r_{3}}, & \text { if } x \in\left[\frac{r_{3}+r_{4}}{2}, r_{4}\right] .\end{cases}
$$

reduced fuzzy variable $\xi$ has the following parametric possibility distribution:

$$
\begin{aligned}
& \mu_{\xi}(x ; \theta, \alpha) \\
& = \begin{cases}\left(1-\theta_{l}+2 \alpha \theta_{l}\right) \frac{x-r_{1}}{r_{2}-r_{1}}, & \text { if } x \in\left[r_{1}, \frac{r_{1}+r_{2}}{2}\right] \\
\frac{\left(1+\theta_{l}-2 \alpha \theta_{l}\right) x-(1-2 \alpha) \theta_{l} r_{2}-r_{1}}{r_{2}-r_{1}}, & \text { if } x \in\left[\frac{r_{1}+r_{2}}{2}, r_{2}\right] \\
1, & \text { if } x \in\left[r_{2}, r_{3}\right] \\
\frac{-\left(1+\theta_{l}-2 \alpha \theta_{l}\right) x+(1-2 \alpha) \theta_{l} r_{3}+r_{4}}{r_{4}-r_{3}}, & \text { if } x \in\left[r_{3}, \frac{r_{3}+r_{4}}{2}\right] \\
\left(1-\theta_{l}+2 \alpha \theta_{l}\right) \frac{r_{4}-x}{r_{4}-r_{3}}, & \text { if } x \in\left[\frac{r_{3}+r_{4}}{2}, r_{4}\right] .\end{cases}
\end{aligned}
$$

If $\alpha \in(0.5,1]$, then the CVaR reduced fuzzy variable $\xi$ has the following parametric possibility distribution:
Theorem 1. Let $\tilde{\xi}=\left(\widetilde{r}_{1}, \widetilde{r}_{2}, \widetilde{r}_{3}, \widetilde{r}_{4} ; \theta_{l}, \theta_{r}\right)$ be a type-2 trapezoidal fuzzy variable, and $\theta=\left(\theta_{l}, \theta_{r}\right)$.

(i) If $\alpha \in(0,0.5]$, the optimistic value of the CVaR reduced fuzzy variable $\xi$ has the following parametric possibility distribution:

$\xi_{\text {sup }}(\beta ; \theta, \alpha)$

$= \begin{cases}r_{4}-\frac{2 \beta}{1-\theta_{1}+2 \alpha \theta_{l}}\left(r_{4}-r_{3}\right), & \beta \in\left[0, \frac{1-\theta_{l}+2 \alpha \theta_{l}}{4}\right] \\ r_{3}+\frac{1-2 \beta}{1+\theta_{l}-2 \alpha \theta_{l}}\left(r_{4}-r_{3}\right), & \beta \in\left[\frac{1-\theta_{l}+2 \alpha \theta_{l}}{4}, \frac{1}{2}\right] \\ r_{2}-\frac{2 \beta-1}{1+\theta_{l}-2 \alpha \theta_{l}}\left(r_{2}-r_{1}\right), & \beta \in\left(\frac{1}{2}, \frac{3+\theta_{l}-2 \alpha \theta_{l}}{4}\right] \\ r_{1}+\frac{2-2 \beta}{1-\theta_{l}+2 \alpha \theta_{l}}\left(r_{2}-r_{1}\right), & \beta \in\left[\frac{3+\theta_{l}-2 \alpha \theta_{l}}{4}, 1\right] .\end{cases}$

(ii) If $\alpha \in(0.5,1]$, the optimistic value of the CVaR reduced fuzzy variable $\xi$ has the following parametric possibility distribution:

$\xi_{\text {sup }}(\beta ; \theta, \alpha)$

$= \begin{cases}r_{4}-\frac{2 \beta}{1-\theta_{r}+2 \alpha \theta_{r}}\left(r_{4}-r_{3}\right), & \beta \in\left[0, \frac{1-\theta_{r}+2 \alpha \theta_{r}}{4}\right] \\ r_{3}+\frac{1-2 \beta}{1+\theta_{r}-2 \alpha \theta_{r}}\left(r_{4}-r_{3}\right), & \beta \in\left[\frac{1-\theta_{r}+2 \alpha \theta_{r}}{4}, \frac{1}{2}\right] \\ r_{2}-\frac{2 \beta-1}{1+\theta_{r}-2 \alpha \theta_{r}}\left(r_{2}-r_{1}\right), & \beta \in\left(\frac{1}{2}, \frac{3+\theta_{r}-2 \alpha \theta_{r}}{4}\right] \\ r_{1}+\frac{2-2 \beta}{1-\theta_{r}+2 \alpha \theta_{r}}\left(r_{2}-r_{1}\right), & \beta \in\left[\frac{3+\theta_{r}-2 \alpha \theta_{r}}{4}, 1\right] .\end{cases}$ 
Note that $\mu_{\xi}\left(\left(r_{1}+r_{2}\right) / 2\right)=\left(3+\theta_{l}-2 \alpha \theta_{l}\right) / 4$ and $\mu_{\xi}\left(\left(r_{3}+\right.\right.$ $\left.\left.r_{4}\right) / 2\right)=\left(1-\theta_{l}+2 \alpha \theta_{l}\right) / 4$. When $\beta \in\left(0,\left(1-\theta_{l}+2 \alpha \theta_{l}\right) / 4\right)$, $\xi_{\text {sup }}(\beta ; \theta, \alpha)$ is the solution of the following equation:

$$
\left(1-\theta_{l}+2 \alpha \theta_{l}\right) \frac{r_{4}-x}{2\left(r_{4}-r_{3}\right)}=\beta
$$

By solving the above equation, we have

$$
\xi_{\text {sup }}(\beta ; \theta, \alpha)=r_{4}-\frac{2 \beta}{1-\theta_{l}+2 \alpha \theta_{l}}\left(r_{4}-r_{3}\right) \text {. }
$$

As for $\beta \in\left(\left(1-\theta_{l}+2 \alpha \theta_{l}\right) / 4,1 / 2\right),\left(1 / 2,\left(3+\theta_{l}-2 \alpha \theta_{l}\right) / 4\right)$, and $\left(\left(3+\theta_{l}-2 \alpha \theta_{l}\right) / 4,1\right)$, it is similar to deduce

$$
\begin{aligned}
& \xi_{\text {sup }}(\beta ; \theta, \alpha)=r_{3}+\frac{1-2 \beta}{1+\theta_{l}-2 \alpha \theta_{l}}\left(r_{4}-r_{3}\right), \\
& \xi_{\text {sup }}(\beta ; \theta, \alpha)=r_{2}-\frac{2 \beta-1}{1+\theta_{l}-2 \alpha \theta_{l}}\left(r_{2}-r_{1}\right), \\
& \xi_{\text {sup }}(\beta ; \theta, \alpha)=r_{1}+\frac{2-2 \beta}{1-\theta_{l}+2 \alpha \theta_{l}}\left(r_{2}-r_{1}\right) .
\end{aligned}
$$

Hence, we have

$$
\begin{aligned}
& \xi_{\text {sup }}(\beta ; \theta, \alpha) \\
& = \begin{cases}r_{4}-\frac{2 \beta}{1-\theta_{l}+2 \alpha \theta_{l}}\left(r_{4}-r_{3}\right), & \beta \in\left[0, \frac{1-\theta_{l}+2 \alpha \theta_{l}}{4}\right] \\
r_{3}+\frac{1-2 \beta}{1+\theta_{l}-2 \alpha \theta_{l}}\left(r_{4}-r_{3}\right), & \beta \in\left[\frac{1-\theta_{l}+2 \alpha \theta_{l}}{4}, \frac{1}{2}\right] \\
r_{2}-\frac{2 \beta-1}{1+\theta_{l}-2 \alpha \theta_{l}}\left(r_{2}-r_{1}\right), & \beta \in\left(\frac{1}{2}, \frac{3+\theta_{l}-2 \alpha \theta_{l}}{4}\right] \\
r_{1}+\frac{2-2 \beta}{1-\theta_{l}+2 \alpha \theta_{l}}\left(r_{2}-r_{1}\right), & \beta \in\left[\frac{3+\theta_{l}-2 \alpha \theta_{l}}{4}, 1\right] .\end{cases}
\end{aligned}
$$

The proof of the assertion (i) is complete.

Theorem 2. Let $\tilde{\xi}=\left(\widetilde{r}_{1}, \widetilde{r}_{2}, \widetilde{r}_{3}, \widetilde{r}_{4} ; \theta_{l}, \theta_{r}\right)$ be a type-2 trapezoidal fuzzy variable, and $\theta=\left(\theta_{l}, \theta_{r}\right)$.

(i) If $\alpha \in(0,0.5]$, the pessimistic value of the CVaR reduced fuzzy variable $\xi$ has the following parametric possibility distribution:

$$
\begin{aligned}
& \xi_{\text {inf }}(\beta ; \theta, \alpha) \\
& = \begin{cases}r_{1}+\frac{2 \beta}{1-\theta_{l}+2 \alpha \theta_{l}}\left(r_{2}-r_{1}\right), & \beta \in\left[0, \frac{1-\theta_{l}+2 \alpha \theta_{l}}{4}\right] \\
r_{2}-\frac{1-2 \beta}{1+\theta_{l}-2 \alpha \theta_{l}}\left(r_{2}-r_{1}\right), & \beta \in\left[\frac{1-\theta_{l}+2 \alpha \theta_{l}}{4}, \frac{1}{2}\right] \\
r_{3}+\frac{2 \beta-1}{1+\theta_{l}-2 \alpha \theta_{l}}\left(r_{4}-r_{3}\right), & \beta \in\left(\frac{1}{2}, \frac{3+\theta_{l}-2 \alpha \theta_{l}}{4}\right] \\
r_{4}-\frac{2-2 \beta}{1-\theta_{l}+2 \alpha \theta_{l}}\left(r_{4}-r_{3}\right), & \beta \in\left[\frac{3+\theta_{l}-2 \alpha \theta_{l}}{4}, 1\right] .\end{cases}
\end{aligned}
$$

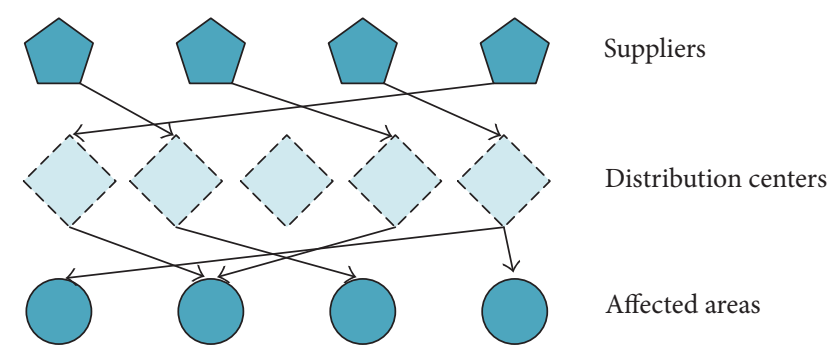

FIGURE 1: An example for emergency network structure.

(ii) If $\alpha \in(0.5,1]$, the pessimistic value of the CVaR reduced fuzzy variable $\xi$ has the following parametric possibility distribution:

$$
\begin{aligned}
& \xi_{\text {inf }}(\beta ; \theta, \alpha) \\
& = \begin{cases}r_{1}+\frac{2 \beta}{1-\theta_{r}+2 \alpha \theta_{r}}\left(r_{2}-r_{1}\right), & \beta \in\left[0, \frac{1-\theta_{r}+2 \alpha \theta_{r}}{4}\right] \\
r_{2}-\frac{1-2 \beta}{1+\theta_{r}-2 \alpha \theta_{r}}\left(r_{2}-r_{1}\right), & \beta \in\left[\frac{1-\theta_{r}+2 \alpha \theta_{r}}{4}, \frac{1}{2}\right] \\
r_{3}+\frac{2 \beta-1}{1+\theta_{r}-2 \alpha \theta_{r}}\left(r_{4}-r_{3}\right), & \beta \in\left(\frac{1}{2}, \frac{3+\theta_{r}-2 \alpha \theta_{r}}{4}\right] \\
r_{4}-\frac{2-2 \beta}{1-\theta_{r}+2 \alpha \theta_{r}}\left(r_{4}-r_{3}\right), & \beta \in\left[\frac{3+\theta_{r}-2 \alpha \theta_{r}}{4}, 1\right] .\end{cases}
\end{aligned}
$$

Proof. It can be proved similarly as Theorem 1 .

\section{Formulation of Prepositioning Emergency Supplies Model}

3.1. Assumptions and Notations. To model our prepositioning emergency supplies problem, we make the following assumptions.

(i) The emergency supply chain is composed of some entities, mainly including suppliers, distribution centers, and affected areas. That is to say, the whole emergency network involves three levels of hierarchical structure, which is shown in Figure 1.

(ii) Each emergency distribution center can be opened in only one of three possible size categories: small, medium, and large, subject to the related fixed cost and maximal capacity.

(iii) There is more than one kind of emergency supplies considered, and each relief is associated with a fixed volume, procurement cost, and transportation cost.

(iv) The supply capacity of supplier, storage capacity of distribution center, demand amount of affected area, and unit procurement cost and transportation cost of emergency relief are uncertain prior to an emergency event. Different from the existing work [9], these uncertain parameters are characterized by variable possibility distributions.

In addition, we adopt the notations displayed in Notations to build our optimization model. 
3.2. Maximal Chance Objective. The prepositioning emergency supplies problem determines the location and size category of a distribution center as well as the quantities and allocations of the prepositioned emergency supplies with the aim of the least total cost. The total cost usually can be expressed as follows:

$$
\begin{aligned}
& f(\mathbf{w}, \mathbf{x}, \mathbf{y}, \mathbf{z} ; \boldsymbol{\xi}, \boldsymbol{\eta}, \zeta)=\sum_{j \in J} \sum_{l \in L} g_{j l} w_{j l}+\sum_{i \in I} \sum_{j \in J} \sum_{m \in M} a_{i m} x_{i j m} \\
& +\sum_{i \in I} \sum_{j \in J} \sum_{m \in M} b_{i j m} x_{i j m}+r\left(\sum_{i \in I} \sum_{j \in J} \sum_{m \in M} \widetilde{\xi}_{i m} y_{i j m}\right. \\
& \left.\quad+\sum_{i \in I} \sum_{j \in J} \sum_{m \in M} \tilde{\eta}_{i j m} y_{i j m}+\sum_{j \in J} \sum_{k \in K} \sum_{m \in M} \widetilde{\zeta}_{j k m} z_{j k m}\right),
\end{aligned}
$$

where $\mathbf{w}=\left\{w_{j l}\right\}, \mathbf{x}=\left\{x_{i j m}\right\}, \mathbf{y}=\left\{y_{i j m}\right\}, \mathbf{z}=\left\{z_{j k m}\right\}, \boldsymbol{\xi}=\left\{\widetilde{\xi}_{i m}\right\}$, $\boldsymbol{\eta}=\left\{\tilde{\eta}_{i j m}\right\}$, and $\boldsymbol{\zeta}=\left\{\widetilde{\zeta}_{j k m}\right\} ; r(\cdot)$ is the CVaR reduced fuzzy variable of type-2 fuzzy variables' linear combination given in the parenthesis. In the right hand of (13), the first one is the fixed cost of opening the distribution centers, the second and third ones are acquisition cost and transportation cost for predisaster emergency supplies, and the last three ones are acquisition cost and transportation cost for postdisaster emergency supplies.

Our objective is to minimize the total cost incurred in the whole process. We adopt the following risk-averse criterion to construct the objective function. Evidently, $f(\mathbf{w}, \mathbf{x}, \mathbf{y}, \mathbf{z} ; \boldsymbol{\xi}, \boldsymbol{\eta}, \boldsymbol{\zeta})$ is a fuzzy variable. For a prescribed real number $f_{0}$, we denote $\operatorname{Cr}\left\{f(\mathbf{w}, \mathbf{x}, \mathbf{y}, \mathbf{z} ; \boldsymbol{\xi}, \boldsymbol{\eta}, \boldsymbol{\zeta}) \leq f_{0}\right\}$ as the credibility distribution of $f(\mathbf{w}, \mathbf{x}, \mathbf{y}, \mathbf{z} ; \boldsymbol{\xi}, \boldsymbol{\eta}, \boldsymbol{\zeta})$. In proposed prepositioning model, we consider maximizing the $\mathrm{Cr}$ of the total cost $f(\mathbf{w}, \mathbf{x}, \mathbf{y}, \mathbf{z} ; \boldsymbol{\xi}, \boldsymbol{\eta}, \boldsymbol{\zeta})$, that is,

$$
\max \operatorname{Cr}\left\{f(\mathbf{w}, \mathbf{x}, \mathbf{y}, \mathbf{z} ; \boldsymbol{\xi}, \boldsymbol{\eta}, \zeta) \leq f_{0}\right\}
$$

By introducing an additional variable $\beta$, the maximal chance objective is equivalent to the following representation:

$$
\begin{array}{ll}
\max & \beta \\
\text { s.t. } & \operatorname{Cr}\left\{f(\mathbf{w}, \mathbf{x}, \mathbf{y}, \mathbf{z} ; \boldsymbol{\xi}, \boldsymbol{\eta}, \boldsymbol{\zeta}) \leq f_{0}\right\} \geq \beta,
\end{array}
$$

which implies that the total cost is less than or equal to $f_{0}$ with credibility at least $\beta(0<\beta \leq 1)$.

3.3. Credibility Constraints. An efficient prepositioning strategy should react effectively in response to the demands of affected areas, in addition to cost-effective operations. Since the exact demands are not known in advance, we describe them by variable possibility distributions. If the demands of victims can be satisfied completely, then the requirement is represented as

$$
\sum_{j \in J} z_{j k m} \geq d_{k m}, \quad \forall k \in K, m \in M,
$$

where $d_{k m}$ is the CVaR reduced fuzzy variable of type-2 fuzzy variable $\widetilde{d}_{k m}$. In practice, however, the assumption above is a challenging requirement for the decision makers due to a large number of complicated constraints. In this case, the demands of affected areas may be satisfied partly. The service quality constraint allows us to provide a predetermined service level $\phi_{k m} \in(0,1)$ to meet the demands in the network [30]. In fuzzy optimization theory, we can address the service quality by the following credibility constraint:

$$
\operatorname{Cr}\left\{\sum_{j \in J} z_{j k m} \geq d_{k m}\right\} \geq \phi_{k m}, \quad \forall k \in K, m \in M
$$

On the other hand, since it is difficult to predict the magnitude of any disaster and subsequent impact on the transportation system, we treat the allocation of emergency supplies in a fuzzy manner. The uncertainty coming from the vulnerability of the transportation network leads to fuzzy capacities, which are characterized by variable possibility distributions. Without loss of generality, it is required that the capacity of supplier cannot be exceeded; that is,

$$
\sum_{j \in J} y_{i j m} \leq \lambda_{i m} S_{i m}, \quad \forall i \in I, m \in M
$$

where $\lambda_{i m}$ is the CVaR reduced fuzzy variable of type-2 fuzzy variable $\tilde{\lambda}_{i m}$. Given a confidence level $\psi_{i m}$, the capacity constraint of supplier can be expressed as follows:

$$
\operatorname{Cr}\left\{\sum_{j \in J} y_{i j m} \leq \lambda_{i m} S_{i m}\right\} \geq \psi_{i m}, \quad \forall i \in I, m \in M
$$

Similarly, it is required that the capacity of distribution center cannot be exceeded; that is,

$$
\sum_{i \in I}\left(\delta_{j m} x_{i j m}+y_{i j m}\right) \geq \sum_{k \in K} z_{j k m}, \quad \forall j \in J, m \in M,
$$

where $\delta_{j m}$ is the CVaR reduced fuzzy variable of type-2 fuzzy variable $\widetilde{\delta}_{j m}$. Given a confidence level $\kappa_{j m}$, the capacity constraint of distribution center can be expressed as follows:

$$
\begin{aligned}
\operatorname{Cr}\left\{\sum_{i \in I}\left(\delta_{j m} x_{i j m}+y_{i j m}\right) \geq \sum_{k \in K} z_{j k m}\right\} & \geq \kappa_{j m}, \\
& \forall j \in J, m \in M .
\end{aligned}
$$

3.4. Fuzzy Prepositioning Emergency Supplies Model. On the basis of the discussion above, we formally construct the 
following maximal chance prepositioning optimization model for disaster management:

$$
\begin{array}{ll}
\max & \beta \\
\text { s.t. } & \operatorname{Cr}\left\{f(\mathbf{w}, \mathbf{x}, \mathbf{y}, \mathbf{z} ; \boldsymbol{\xi}, \boldsymbol{\eta}, \boldsymbol{\zeta}) \leq f_{0}\right\} \geq \beta \\
& \sum_{l \in L} w_{j l} \leq 1, \quad \forall j \in J \\
& \sum_{i \in I} y_{i j m} \leq Q \sum_{l \in L} w_{j l}, \quad \forall j \in J, m \in M \\
& \sum_{k \in K} z_{j k m} \leq Q \sum_{l \in L} w_{j l}, \quad \forall j \in J, m \in M \\
& \sum_{j \in J} x_{i j m} \leq S_{i m}, \quad \forall i \in I, m \in M \\
& \sum_{i \in I} \sum_{m \in M} c_{m} x_{i j m} \leq \sum_{l \in L} v_{l} w_{j l}, \quad \forall j \in J \\
& \operatorname{Cr}\left\{\sum_{j \in J} z_{j k m} \geq d_{k m}\right\} \geq \phi_{k m},
\end{array}
$$$$
\forall k \in K, m \in M
$$$$
\operatorname{Cr}\left\{\sum_{j \in J} y_{i j m} \leq \lambda_{i m} S_{i m}\right\} \geq \psi_{i m}
$$$$
\forall i \in I, m \in M
$$$$
\operatorname{Cr}\left\{\sum_{i \in I}\left(\delta_{j m} x_{i j m}+y_{i j m}\right) \geq \sum_{k \in K} z_{j k m}\right\} \geq \kappa_{j m},
$$$$
\forall j \in J, m \in M
$$$$
w_{j l} \in\{0,1\}, \quad \forall j \in J, l \in L
$$$$
x_{i j m}, y_{i j m}, z_{j k m} \geq 0 \text {, }
$$$$
\forall i \in I, j \in J, k \in K, m \in M .
$$

The objective function (22) is to maximize the credibility $\beta$ of the total cost in the sense of (23). Constraint (23) means that the total cost is less than or equal to the predetermined value $f_{0}$ with credibility at least $\beta$. Constraint (24) limits the number of open distribution centers at node $j$ to no more than one. If a distribution center is made available at location $j$, various reliefs can be stocked there. Constraints (25) and (26) require that each commodity prepositioned is assigned to the open facilities and the allocation does not exceed the facility capacity. Constraints (27) and (28) imply the capacity limitation of supplier and distribution center before disaster. Constraint (29) imposes the amount of commodity $k$ for affected area $k$ should meet the demand with a given service level $\phi_{k m}$. Uncertainty resulting from the vulnerability of the transportation system leads to fuzzy capacities. So after disaster the acquisition and allocation cannot surpass the supplier's and distribution center's capacity with the respective predetermined credibility $\psi_{i m}$ and $\kappa_{j m}$, which can be written in constraints (30) and (31). Constraint (32) emphasizes that $w_{j l}$ equals 1 if there is a distribution center with size category $l$ located at node $j$, and 0 otherwise. Finally, constraint (33) ensures the nonnegativity of acquisition variables and allocation variables.

\section{Solution Method}

In order to solve our prepositioning model (22)-(33), we require turning credibility constraints into their equivalent deterministic forms. In this section, we discuss this issue in detail.

In our prepositioning emergency supplies model, the parameter $b_{i j m}$ is the transportation cost for emergency relief $m$ from supplier $i$ to distribution center $j$. It can be expressed as $b_{i j m}=e_{i j} b_{m}$, where $b_{m}$ is the unit transportation cost for commodity $m$ before disaster and $e_{i j}$ represents the distance between supplier $i$ and distribution center $j$ in normal state. The parameter $\widetilde{\eta}_{i j m}$ is the type- 2 fuzzy transportation cost for emergency relief $m$ purchased by supplier $i$ and transported to distribution center $j$. It can be expressed as $\widetilde{\eta}_{i j m}=e_{i j} \widetilde{\eta}_{m}$, where $\widetilde{\eta}_{m}$ is the unit transportation cost for commodity $m$ after disaster. Similarly, the parameter $\widetilde{\zeta}_{j k m}$ can be expressed as $\widetilde{\zeta}_{j k m}=E_{j k} \widetilde{\zeta}_{m}$. In addition, the parameter $\widetilde{\lambda}_{i m}$ is the type-2 fuzzy proportion of stocked material for emergency relief $m$ at supplier $i$ after disaster. It can be expressed as $\widetilde{\lambda}_{i m}=p_{m} \widetilde{\lambda}_{i}$, where $p_{m}$ and $\tilde{\lambda}_{i}$ are the relative quality level of emergency relief $m$ and the damage level of supplier $i$, respectively. The parameter $\widetilde{\delta}_{j m}$ can be expressed as $\widetilde{\delta}_{j m}=p_{m} \widetilde{\delta}_{j}$.

We now represent all type- 2 fuzzy variables as the functions of $\widetilde{\eta}_{1}, \ldots, \widetilde{\eta}_{|M|}, \widetilde{\zeta}_{1}, \ldots, \widetilde{\zeta}_{|M|}, \widetilde{\lambda}_{1}, \ldots, \widetilde{\lambda}_{|I|}$, and $\widetilde{\delta}_{1}, \ldots, \widetilde{\delta}_{|J|}$, except for type-2 fuzzy variables $\tilde{\xi}_{i m}$ and $\tilde{d}_{k m}$. The number of fuzzy variables is $|I|+|J|+(|I|+|J|+2)|M|$. In general, the possibility distributions of these fuzzy parameters may have different shapes, but trapezoidal membership functions are most frequently used in practice. Without loss of generality, we assume in our prepositioning model that the whole type-2 fuzzy variables are characterized by type- 2 trapezoidal fuzzy variables. These type- 2 fuzzy variables are independent in the sense of [20]. Furthermore, their elements are defined by

$$
\begin{aligned}
\tilde{\xi}_{i m} & =\left(r_{1, i m}^{\xi}, r_{2, i m}^{\xi}, r_{3, i m}^{\xi}, r_{4, i m}^{\xi} ; \theta_{l, i m}^{\xi}, \theta_{r, i m}^{\xi}\right), \\
\tilde{d}_{k m} & =\left(r_{1, k m}^{d}, r_{2, k m}^{d}, r_{3, k m}^{d}, r_{4, k m}^{d} ; \theta_{l, k m}^{d}, \theta_{r, k m}^{d}\right), \\
\tilde{\eta}_{m} & =\left(r_{1, m}^{\eta}, r_{2, m}^{\eta}, r_{3, m}^{\eta}, r_{4, m}^{\eta} ; \theta_{l, m}^{\eta}, \theta_{r, m}^{\eta}\right), \\
\tilde{\zeta}_{m} & =\left(r_{1, m}^{\zeta}, r_{2, m}^{\zeta}, r_{3, m}^{\zeta}, r_{4, m}^{\zeta} ; \theta_{l, m}^{\zeta}, \theta_{r, m}^{\zeta}\right), \\
\tilde{\lambda}_{i} & =\left(r_{1, i}^{\lambda}, r_{2, i}^{\lambda}, r_{3, i}^{\lambda}, r_{4, i}^{\lambda}, \theta_{l, i}^{\lambda}, \theta_{r, i}^{\lambda}\right), \\
\tilde{\delta}_{j} & =\left(r_{1, j}^{\delta}, r_{2, j}^{\delta}, r_{3, j}^{\delta}, r_{4, j}^{\delta} ; \theta_{l, j}^{\delta}, \theta_{r, j}^{\delta}\right) .
\end{aligned}
$$

4.1. Equivalent Forms of Objective and Constraints. The following theorems discuss the equivalent deterministic representations of credibility constraints in prepositioning model (22)-(33). 
We first consider the analytical expression of objective function $\beta$ included in cost constraint (23); that is,

$$
\operatorname{Cr}\left\{f(\mathbf{w}, \mathbf{x}, \mathbf{y}, \mathbf{z} ; \boldsymbol{\xi}, \boldsymbol{\eta}, \boldsymbol{\zeta}) \leq f_{0}\right\} \geq \beta
$$

where $f(\mathbf{w}, \mathbf{x}, \mathbf{y}, \mathbf{z} ; \boldsymbol{\xi}, \boldsymbol{\eta}, \boldsymbol{\zeta})$ can refer to (13).

Theorem 3. Consider cost constraint (23) in prepositioning model (22)-(33). Let the acquisition cost $\widetilde{\xi}_{i m}=\left(r_{1, i m}^{\xi}, r_{2, i m}^{\xi}\right.$, $\left.r_{3, i m}^{\xi}, r_{4, i m}^{\xi} ; \theta_{l, i m}^{\xi}, \theta_{r, i m}^{\xi}\right)$, unit transportation cost $\tilde{\eta}_{m}=\left(r_{1, m}^{\eta}\right.$, $\left.r_{2, m}^{\eta}, r_{3, m}^{\eta}, r_{4, m}^{\eta} ; \theta_{l, m}^{\eta}, \theta_{r, m}^{\eta}\right)$, and $\tilde{\zeta}_{m}=\left(r_{1, m}^{\zeta}, r_{2, m}^{\zeta}, r_{3, m}^{\zeta}, r_{4, m}^{\zeta} ; \theta_{l, m}^{\zeta}\right.$, $\left.\theta_{r, m}^{\zeta}\right)$ be mutually independent type-2 trapezoidal fuzzy variables. Suppose that

$$
\begin{aligned}
& r\left(\sum_{i \in I} \sum_{j \in J} \sum_{m \in M} \widetilde{\xi}_{i m} y_{i j m}+\sum_{i \in I} \sum_{j \in J} \sum_{m \in M} e_{i j} \widetilde{\eta}_{m} y_{i j m}\right. \\
& \left.\quad+\sum_{j \in J} \sum_{k \in K} \sum_{m \in M} E_{j k} \widetilde{\zeta}_{m} z_{j k m}\right)
\end{aligned}
$$

is the CVaR reduced fuzzy variable of type-2 fuzzy variables' linear combination given in the parenthesis in case of $\alpha \in$ $[0.5,1]$. If one denotes

$$
\begin{aligned}
& r_{1}= \sum_{i \in I} \sum_{j \in J} \sum_{m \in M} r_{1, i m}^{\xi} y_{i j m}+\sum_{i \in I} \sum_{j \in J} \sum_{m \in M} e_{i j} r_{1, m}^{\eta} y_{i j m} \\
&+\sum_{j \in J} \sum_{k \in K} \sum_{m \in M} E_{j k} r_{1, m}^{\zeta} z_{j k m}, \\
& r_{2}= \sum_{i \in I} \sum_{j \in J} \sum_{m \in M} r_{2, i m}^{\xi} y_{i j m}+\sum_{i \in I} \sum_{j \in J} \sum_{m \in M} e_{i j} r_{2, m}^{\eta} y_{i j m} \\
&+\sum_{j \in J} \sum_{k \in K} \sum_{m \in M} E_{j k} r_{2, m}^{\zeta} z_{j k m}, \\
& r_{3}= \sum_{i \in} \sum_{j \in J} \sum_{m \in M} r_{3, i m}^{\xi} y_{i j m}+\sum_{i \in I} \sum_{j \in J} \sum_{m \in M} e_{i j} r_{3, m}^{\eta} y_{i j m} \\
&+\sum_{j \in J} \sum_{k \in K} \sum_{m \in M} E_{j k} r_{3, m}^{\zeta} z_{j k m}, \\
& \theta_{r}= \min \left\{\theta_{l, i m}^{\xi}, \theta_{l, m}^{\eta}, \theta_{l, m}^{\zeta} \mid \forall i \in I, m \in M\right\}, \\
& r_{4}= \sum_{i \in I} \sum_{j \in J} \sum_{m \in M} r_{4, i m}^{\xi} y_{i j m}+\sum_{i \in I} \sum_{j \in J} \sum_{m \in M} e_{i j} r_{4, m}^{\eta} y_{i j m} \\
&+\sum_{j \in J} \sum_{k \in K} \sum_{m \in M} E_{j k} r_{4, m}^{\zeta} z_{j k m}, \\
&\left\{\theta_{l, i m}^{\xi}, \theta_{l, m}^{\eta}, \theta_{l, m}^{\zeta} \mid \forall i \in I, m \in M\right\}, \\
&
\end{aligned}
$$

then cost constraint (23) is equivalent to

$$
\begin{aligned}
& r\left(\sum_{i \in I} \sum_{j \in J} \sum_{m \in M} \widetilde{\xi}_{i m} y_{i j m}+\sum_{i \in I} \sum_{j \in J} \sum_{m \in M} e_{i j} \widetilde{\eta}_{m} y_{i j m}\right. \\
& \left.\quad+\sum_{j \in J} \sum_{k \in K} \sum_{m \in M} E_{j k} \widetilde{\zeta}_{m} z_{j k m}\right)_{i n f}(\beta) \leq f_{0} \\
& \quad-\sum_{j \in J} \sum_{l \in L} g_{j l} w_{j l}-\sum_{i \in I} \sum_{j \in J} \sum_{m \in M} a_{i m} x_{i j m} \\
& \quad-\sum_{i \in I} \sum_{j \in J} \sum_{m \in M} b_{i j m} x_{i j m},
\end{aligned}
$$

where

$$
\begin{aligned}
& r\left(\sum_{i \in I} \sum_{j \in J} \sum_{m \in M} \tilde{\xi}_{i m} y_{i j m}+\sum_{i \in I} \sum_{j \in J} \sum_{m \in M} e_{i j} \widetilde{\eta}_{m} y_{i j m}\right. \\
& \left.\quad+\sum_{j \in J} \sum_{k \in K} \sum_{m \in M} E_{j k} \widetilde{\zeta}_{m} z_{j k m}\right)_{\text {inf }}(\beta) \\
& \quad= \begin{cases}r_{1}+\frac{2 \beta}{1-\theta_{r}+2 \alpha \theta_{r}}\left(r_{2}-r_{1}\right), & \beta \in\left[0, \frac{1-\theta_{r}+2 \alpha \theta_{r}}{4}\right] \\
r_{2}-\frac{1-2 \beta}{1+\theta_{r}-2 \alpha \theta_{r}}\left(r_{2}-r_{1}\right), & \beta \in\left[\frac{1-\theta_{r}+2 \alpha \theta_{r}}{4}, \frac{1}{2}\right] \\
r_{3}+\frac{2 \beta-1}{1+\theta_{r}-2 \alpha \theta_{r}}\left(r_{4}-r_{3}\right), & \beta \in\left(\frac{1}{2}, \frac{3+\theta_{r}-2 \alpha \theta_{r}}{4}\right] \\
r_{4}-\frac{2-2 \beta}{1-\theta_{r}+2 \alpha \theta_{r}}\left(r_{4}-r_{3}\right), & \beta \in\left[\frac{3+\theta_{r}-2 \alpha \theta_{r}}{4}, 1\right] .\end{cases}
\end{aligned}
$$

Proof. Since the acquisition cost $\widetilde{\xi}_{i m}=\left(r_{1, i m}^{\xi}, r_{2, i m}^{\xi}, r_{3, i m}^{\xi}\right.$, $\left.r_{4, i m}^{\xi} ; \theta_{l, i m}^{\xi}, \theta_{r, i m}^{\xi}\right)$, unit transportation cost $\tilde{\eta}_{m}=\left(r_{1, m}^{\eta}, r_{2, m}^{\eta}\right.$, $\left.r_{3, m}^{\eta}, r_{4, m}^{\eta} ; \theta_{l, m}^{\eta}, \theta_{r, m}^{\eta}\right)$, and $\widetilde{\zeta}_{m}=\left(r_{1, m}^{\zeta}, r_{2, m}^{\zeta}, r_{3, m}^{\zeta}, r_{4, m}^{\zeta} ; \theta_{l, m}^{\zeta}, \theta_{r, m}^{\zeta}\right)$ are mutually independent type- 2 trapezoidal fuzzy variables, the postdisaster total acquisition and transportation cost

$$
\begin{aligned}
& \sum_{i \in I} \sum_{j \in J} \sum_{m \in M} \tilde{\xi}_{i m} y_{i j m}+\sum_{i \in I} \sum_{j \in J} \sum_{m \in M} e_{i j} \widetilde{\eta}_{m} y_{i j m} \\
& +\sum_{j \in J} \sum_{k \in K} \sum_{m \in M} E_{j k} \widetilde{\zeta}_{m} z_{j k m}
\end{aligned}
$$

is also type-2 trapezoidal fuzzy variable [31], denoted as $\left(r_{1}, r_{2}, r_{3}, r_{4} ; \theta_{l}, \theta_{r}\right)$, where

$$
\begin{aligned}
r_{1}= & \sum_{i \in I} \sum_{j \in J} \sum_{m \in M} r_{1, i m}^{\xi} y_{i j m}+\sum_{i \in I} \sum_{j \in J} \sum_{m \in M} e_{i j} r_{1, m}^{\eta} y_{i j m} \\
& +\sum_{j \in J} \sum_{k \in K} \sum_{m \in M} E_{j k} r_{1, m}^{\zeta} z_{j k m}, \\
r_{2}= & \sum_{i \in I} \sum_{j \in J} \sum_{m \in M} r_{2, i m}^{\xi} y_{i j m}+\sum_{i \in I} \sum_{j \in J} \sum_{m \in M} e_{i j} r_{2, m}^{\eta} y_{i j m} \\
& +\sum_{j \in J} \sum_{k \in K} \sum_{m \in M} E_{j k} r_{2, m}^{\zeta} z_{j k m},
\end{aligned}
$$




$$
\begin{aligned}
r_{3}= & \sum_{i \in I} \sum_{j \in J} \sum_{m \in M} r_{3, i m}^{\xi} y_{i j m}+\sum_{i \in I} \sum_{j \in J} \sum_{m \in M} e_{i j} r_{3, m}^{\eta} y_{i j m} \\
& +\sum_{j \in J} \sum_{k \in K} \sum_{m \in M} E_{j k} r_{3, m}^{\zeta} z_{j k m}, \\
r_{4}= & \sum_{i \in I} \sum_{j \in J} \sum_{m \in M} r_{4, i m}^{\xi} y_{i j m}+\sum_{i \in I} \sum_{j \in J} \sum_{m \in M} e_{i j} r_{4, m}^{\eta} y_{i j m} \\
& +\sum_{j \in J} \sum_{k \in K} \sum_{m \in M} E_{j k} r_{4, m}^{\zeta} z_{j k m}, \\
\theta_{l}= & \max \left\{\theta_{l, i m}^{\xi}, \theta_{l, m}^{\eta}, \theta_{l, m}^{\zeta} \mid \forall i \in I, m \in M\right\}, \\
\theta_{r}= & \min \left\{\theta_{l, i m}^{\xi}, \theta_{l, m}^{\eta}, \theta_{l, m}^{\zeta} \mid \forall i \in I, m \in M\right\} .
\end{aligned}
$$

Suppose that

$$
\begin{aligned}
& r\left(\sum_{i \in I} \sum_{j \in J} \sum_{m \in M} \widetilde{\xi}_{i m} y_{i j m}+\sum_{i \in I} \sum_{j \in J} \sum_{m \in M} e_{i j} \widetilde{\eta}_{m} y_{i j m}\right. \\
& \left.+\sum_{j \in J} \sum_{k \in K} \sum_{m \in M} E_{j k} \widetilde{\zeta}_{m} z_{j k m}\right)
\end{aligned}
$$

is the CVaR reduced fuzzy variable of type-2 fuzzy variables' linear combination given in the parenthesis in case of $\alpha \epsilon$ $[0.5,1]$. So cost constraint (23) is equivalent to

$$
\begin{aligned}
\operatorname{Cr} & \left\{r \left(\sum_{i \in I} \sum_{j \in J} \sum_{m \in M} \tilde{\xi}_{i m} y_{i j m}+\sum_{i \in I} \sum_{j \in J} \sum_{m \in M} \tilde{\eta}_{i j m} y_{i j m}\right.\right. \\
& \left.+\sum_{j \in J} \sum_{k \in K} \sum_{m \in M} \widetilde{\zeta}_{j k m} z_{j k m}\right) \leq f_{0}-\sum_{j \in J} \sum_{l \in L} g_{j l} w_{j l} \\
& \left.-\sum_{i \in I} \sum_{j \in J} \sum_{m \in M} a_{i m} x_{i j m}-\sum_{i \in I} \sum_{j \in J} \sum_{m \in M} b_{i j m} x_{i j m}\right\} \geq \beta ;
\end{aligned}
$$

that is,

$$
\begin{gathered}
r\left(\sum_{i \in I} \sum_{j \in J} \sum_{m \in M} \tilde{\xi}_{i m} y_{i j m}+\sum_{i \in I} \sum_{j \in J} \sum_{m \in M} e_{i j} \tilde{\eta}_{m} y_{i j m}\right. \\
\left.+\sum_{j \in J} \sum_{k \in K} \sum_{m \in M} E_{j k} \widetilde{\zeta}_{m} z_{j k m}\right)_{\mathrm{inf}}(\beta) \leq f_{0}
\end{gathered}
$$

$$
\begin{aligned}
& -\sum_{j \in J \in L} \sum_{l \in L} g_{j l} w_{j l}-\sum_{i \in I} \sum_{j \in J} \sum_{m \in M} a_{i m} x_{i j m} \\
& -\sum_{i \in I} \sum_{j \in J} \sum_{m \in M} b_{i j m} x_{i j m} .
\end{aligned}
$$

Based on Theorem 2, the pessimistic value of the CVaR reduced fuzzy variable has the following parametric possibility distribution:

$$
\begin{aligned}
& r\left(\sum_{i \in I} \sum_{j \in J} \sum_{m \in M} \tilde{\xi}_{i m} y_{i j m}+\sum_{i \in I} \sum_{j \in J} \sum_{m \in M} e_{i j} \tilde{\eta}_{m} y_{i j m}\right. \\
& \left.\quad+\sum_{j \in J} \sum_{k \in K} \sum_{m \in M} E_{j k} \widetilde{\zeta}_{m} z_{j k m}\right)_{\text {inf }}(\beta) \\
& \quad= \begin{cases}r_{1}+\frac{2 \beta}{1-\theta_{r}+2 \alpha \theta_{r}}\left(r_{2}-r_{1}\right), \quad \beta \in\left[0, \frac{1-\theta_{r}+2 \alpha \theta_{r}}{4}\right] \\
r_{2}-\frac{1-2 \beta}{1+\theta_{r}-2 \alpha \theta_{r}}\left(r_{2}-r_{1}\right), & \beta \in\left[\frac{1-\theta_{r}+2 \alpha \theta_{r}}{4}, \frac{1}{2}\right] \\
r_{3}+\frac{2 \beta-1}{1+\theta_{r}-2 \alpha \theta_{r}}\left(r_{4}-r_{3}\right), & \beta \in\left(\frac{1}{2}, \frac{3+\theta_{r}-2 \alpha \theta_{r}}{4}\right] \\
r_{4}-\frac{2-2 \beta}{1-\theta_{r}+2 \alpha \theta_{r}}\left(r_{4}-r_{3}\right), & \beta \in\left[\frac{3+\theta_{r}-2 \alpha \theta_{r}}{4}, 1\right] .\end{cases}
\end{aligned}
$$

The proof of the assertion is complete.

In the following, we deal with the analytical expression of service quality constraint (29); that is,

$$
\operatorname{Cr}\left\{\sum_{j \in J} z_{j k m} \geq d_{k m}\right\} \geq \phi_{k m}, \quad \forall k \in K, m \in M .
$$

Theorem 4. Consider service quality constraint (29) in prepositioning model (22)-(33). Let the demand $\widetilde{d}_{k m}=$ $\left(r_{1, k m}^{d}, r_{2, k m}^{d}, r_{3, k m}^{d}, r_{4, k m}^{d} ; \theta_{l, k m}^{d}, \theta_{r, k m}^{d}\right)$ be a type-2 trapezoidal fuzzy variable. Suppose that $d_{k m}$ is its CVaR reduced fuzzy variables with $\alpha_{k m}^{d} \in[0,0.5]$; then service quality constraint (29) is equivalent to

$$
\sum_{j \in J} z_{j k m} \geq d_{k m, \text { inf }}\left(\phi_{k m}\right),
$$

where

$$
d_{k m, \text { inf }}\left(\phi_{k m}\right)= \begin{cases}r_{1, k m}^{d}+\frac{2 \phi_{k m}}{1-\theta_{l, k m}^{d}+2 \alpha_{k m}^{d} \theta_{l, k m}^{d}}\left(r_{2, k m}^{d}-r_{1, k m}^{d}\right), & \phi_{k m} \in\left[0, \frac{1-\theta_{l, k m}^{d}+2 \alpha_{k m}^{d} \theta_{l, k m}^{d}}{4}\right] \\ r_{2, k m}^{d}-\frac{1-2 \phi_{k m}}{1+\theta_{l, k m}^{d}-2 \alpha_{k m}^{d} \theta_{l, k m}^{d}}\left(r_{2, k m}^{d}-r_{1, k m}^{d}\right), & \phi_{k m} \in\left[\frac{1-\theta_{l, k m}^{d}+2 \alpha_{k m}^{d} \theta_{l, k m}^{d}}{4}, \frac{1}{2}\right] \\ r_{3, k m}^{d}+\frac{2 \phi_{k m}-1}{1+\theta_{l, k m}^{d}-2 \alpha_{k m}^{d} \theta_{l, k m}^{d}}\left(r_{4, k m}^{d}-r_{3, k m}^{d}\right), & \phi_{k m} \in\left(\frac{1}{2}, \frac{3+\theta_{l, k m}^{d}-2 \alpha_{k m}^{d} \theta_{l, k m}^{d}}{4}\right] \\ r_{4, k m}^{d}-\frac{2-2 \phi_{k m}}{1-\theta_{l, k m}^{d}+2 \alpha_{k m}^{d} \theta_{l, k m}^{d}}\left(r_{4, k m}^{d}-r_{3, k m}^{d}\right), & \phi_{k m} \in\left[\frac{3+\theta_{l, k m}^{d}-2 \alpha_{k m}^{d} \theta_{l, k m}^{d}}{4}, 1\right] .\end{cases}
$$


Proof. Since $d_{k m}$ is the CVaR reduced fuzzy variable of $\tilde{d}_{k m}$ in the case of $\alpha_{k m}^{d} \in[0,0.5]$, then service quality constraint (29) is equivalent to
Based on Theorem 2, the pessimistic value of the CVaR reduced fuzzy variable has the following parametric possibility distribution:

$$
\sum_{j \in J} z_{j k m} \geq d_{k m, \text { inf }}\left(\phi_{k m}\right)
$$

$$
d_{k m, \text { inf }}\left(\phi_{k m}\right)= \begin{cases}r_{1, k m}^{d}+\frac{2 \phi_{k m}}{1-\theta_{l, k m}^{d}+2 \alpha_{k m}^{d} \theta_{l, k m}^{d}}\left(r_{2, k m}^{d}-r_{1, k m}^{d}\right), & \phi_{k m} \in\left[0, \frac{1-\theta_{l, k m}^{d}+2 \alpha_{k m}^{d} \theta_{l, k m}^{d}}{4}\right] \\ r_{2, k m}^{d}-\frac{1-2 \phi_{k m}}{1+\theta_{l, k m}^{d}-2 \alpha_{k m}^{d} \theta_{l, k m}^{d}}\left(r_{2, k m}^{d}-r_{1, k m}^{d}\right), & \phi_{k m} \in\left[\frac{1-\theta_{l, k m}^{d}+2 \alpha_{k m}^{d} \theta_{l, k m}^{d}, \frac{1}{2}}{4}\right] \\ r_{3, k m}^{d}+\frac{2 \phi_{k m}-1}{1+\theta_{l, k m}^{d}-2 \alpha_{k m}^{d} \theta_{l, k m}^{d}}\left(r_{4, k m}^{d}-r_{3, k m}^{d}\right), & \phi_{k m} \in\left(\frac{1}{2}, \frac{3+\theta_{l, k m}^{d}-2 \alpha_{k m}^{d} \theta_{l, k m}^{d}}{4}\right] \\ r_{4, k m}^{d}-\frac{2-2 \phi_{k m}}{1-\theta_{l, k m}^{d}+2 \alpha_{k m}^{d} \theta_{l, k m}^{d}}\left(r_{4, k m}^{d}-r_{3, k m}^{d}\right), & \phi_{k m} \in\left[\frac{3+\theta_{l, k m}^{d}-2 \alpha_{k m}^{d} \theta_{l, k m}^{d}}{4}, 1\right] .\end{cases}
$$

The proof of the assertion is complete.

Finally, we derive the analytical expressions of emergency relief's proportion constraints (30) and (31); that is,

$$
\begin{gathered}
\operatorname{Cr}\left\{\sum_{j \in J} y_{i j m} \leq \lambda_{i m} S_{i m}\right\} \geq \psi_{i m}, \\
\quad \forall i \in I, m \in M ; \\
\operatorname{Cr}\left\{\sum_{i \in I}\left(\delta_{j m} x_{i j m}+y_{i j m}\right) \geq \sum_{k \in K} z_{j k m}\right\} \geq \kappa_{j m},
\end{gathered}
$$$$
\forall j \in J, m \in M \text {. }
$$

Theorem 5. Consider emergency relief's proportion constraint (30) for supplier $i$ in prepositioning model (22)-(33). Let the supplier's damage level $\widetilde{\lambda}_{i}=\left(r_{1, i}^{\lambda}, r_{2, i}^{\lambda}, r_{3, i}^{\lambda}, r_{4, i}^{\lambda} ; \theta_{l, i}^{\lambda}, \theta_{r, i}^{\lambda}\right)$ be a type-2 trapezoidal fuzzy variable. Suppose that $\lambda_{i}$ is its CVaR reduced fuzzy variables with $\alpha_{i}^{\lambda} \in[0,0.5]$; then emergency relief's proportion constraint (30) for supplier $i$ is equivalent to

$$
\sum_{j \in J} y_{i j m} \leq \lambda_{i, \sup }\left(\psi_{i m}\right) p_{m} S_{i m}
$$

where

$$
\lambda_{i, \text { sup }}\left(\psi_{i m}\right)= \begin{cases}r_{4, i}^{\lambda}-\frac{2 \psi_{i m}}{1-\theta_{l, i}^{\lambda}+2 \alpha_{i}^{\lambda} \theta_{l, i}^{\lambda}}\left(r_{4, i}^{\lambda}-r_{3, i}^{\lambda}\right), & \psi_{i m} \in\left[0, \frac{1-\theta_{l, i}^{\lambda}+2 \alpha_{i}^{\lambda} \theta_{l, i}^{\lambda}}{4}\right] \\ r_{3, i}^{\lambda}+\frac{1-2 \psi_{i m}}{1+\theta_{l, i}^{\lambda}-2 \alpha_{i}^{\lambda} \theta_{l, i}^{\lambda}}\left(r_{4, i}^{\lambda}-r_{3, i}^{\lambda}\right), & \psi_{i m} \in\left[\frac{1-\theta_{l, i}^{\lambda}+2 \alpha_{i}^{\lambda} \theta_{l, i}^{\lambda}}{4}, \frac{1}{2}\right] \\ r_{2, i}^{\lambda}-\frac{2 \psi_{i m}-1}{1+\theta_{l, i}^{\lambda}-2 \alpha_{i}^{\lambda} \theta_{l, i}^{\lambda}}\left(r_{2, i}^{\lambda}-r_{1, i}^{\lambda}\right), & \psi_{i m} \in\left[\frac{1}{2}, \frac{3+\theta_{l, i}^{\lambda}-2 \alpha_{i}^{\lambda} \theta_{l, i}^{\lambda}}{4}\right] \\ r_{1, i}^{\lambda}+\frac{2-2 \psi_{i m}}{1-\theta_{l, i}^{\lambda}+2 \alpha_{i}^{\lambda} \theta_{l, i}^{\lambda}}\left(r_{2, i}^{\lambda}-r_{1, i}^{\lambda}\right), & \psi_{i m} \in\left[\frac{3+\theta_{l, i}^{\lambda}-2 \alpha_{i}^{\lambda} \theta_{l, i}^{\lambda}}{4}, 1\right] .\end{cases}
$$


Proof. Since $\lambda_{i}$ is the CVaR reduced fuzzy variable of $\tilde{\lambda}_{i}$ in the case of $\alpha_{i}^{\lambda} \in[0,0.5]$, then emergency relief's proportion constraint (30) for supplier $i$ is equivalent to

$$
\sum_{j \in J} y_{i j m} \leq \lambda_{i, \text { sup }}\left(\psi_{i m}\right) p_{m} S_{i m} .
$$

Based on Theorem 1, the optimistic value of the CVaR reduced fuzzy variable has the following parametric possibility distribution:

$$
\lambda_{i, s u p}\left(\psi_{i m}\right)= \begin{cases}r_{4, i}^{\lambda}-\frac{2 \psi_{i m}}{1-\theta_{l, i}^{\lambda}+2 \alpha_{i}^{\lambda} \theta_{l, i}^{\lambda}}\left(r_{4, i}^{\lambda}-r_{3, i}^{\lambda}\right), & \psi_{i m} \in\left[0, \frac{1-\theta_{l, i}^{\lambda}+2 \alpha_{i}^{\lambda} \theta_{l, i}^{\lambda}}{4}\right] \\ r_{3, i}^{\lambda}+\frac{1-2 \psi_{i m}}{1+\theta_{l, i}^{\lambda}-2 \alpha_{i}^{\lambda} \theta_{l, i}^{\lambda}}\left(r_{4, i}^{\lambda}-r_{3, i}^{\lambda}\right), & \psi_{i m} \in\left[\frac { 1 - \theta _ { l , i } ^ { \lambda } + 2 \alpha _ { i } ^ { \lambda } \theta _ { l , i } ^ { \lambda } , \frac { 1 } { 2 } ] } { 4 } \left(\psi_{i m}^{\lambda}-1\right.\right. \\ r_{2, i}^{\lambda}-\frac{2 \psi_{i m}^{\lambda}}{1+\theta_{l, i}^{\lambda}-2 \alpha_{i}^{\lambda} \theta_{l, i}^{\lambda}}\left(r_{2, i}^{\lambda}-r_{1, i}^{\lambda}\right), & \psi_{i m} \in\left(\frac{1}{2}, \frac{3+\theta_{l, i}^{\lambda}-2 \alpha_{i}^{\lambda} \theta_{l, i}^{\lambda}}{4}\right] \\ r_{1, i}^{\lambda}+\frac{2-2 \psi_{i m}^{\lambda}}{1-\theta_{l, i}^{\lambda}+2 \alpha_{i}^{\lambda} \theta_{l, i}^{\lambda}}\left(r_{2, i}^{\lambda}-r_{1, i}^{\lambda}\right), & \psi_{i m} \in\left[\frac{3+\theta_{l, i}^{\lambda}-2 \alpha_{i}^{\lambda} \theta_{l, i}^{\lambda}}{4}, 1\right] .\end{cases}
$$

The proof of the assertion is complete.

Theorem 6. Consider emergency relief's proportion constraint (31) for distribution center $j$ in prepositioning model (22)-(33). Let the distribution center's damage level $\widetilde{\delta}_{j}=$ $\left(r_{1, j}^{\delta}, r_{2, j}^{\delta}, r_{3, j}^{\delta}, r_{4, j}^{\delta} ; \theta_{l, j}^{\delta}, \theta_{r, j}^{\delta}\right)$ be a type-2 trapezoidal fuzzy variable. Suppose that $\delta_{j}$ is its CVaR reduced fuzzy variables with $\alpha_{j}^{\delta} \in[0,0.5]$; then emergency relief's proportion constraint (31) for distribution center $j$ is equivalent to

$$
\sum_{k \in K} z_{j k m}-\sum_{j \in J} y_{i j m} \leq \sum_{i \in I} \delta_{j, \text { sup }}\left(\kappa_{j m}\right) p_{m} x_{i j m}
$$

where

$$
\delta_{j, \text { sup }}\left(\kappa_{j m}\right)= \begin{cases}r_{4, j}^{\delta}-\frac{2 \kappa_{j m}}{1-\theta_{l, j}^{\delta}+2 \alpha_{j}^{\delta} \theta_{l, j}^{\delta}}\left(r_{4, j}^{\delta}-r_{3, j}^{\delta}\right), & \kappa_{j m} \in\left[0, \frac{1-\theta_{l, j}^{\delta}+2 \alpha_{j}^{\delta} \theta_{l, j}^{\delta}}{4}\right] \\ r_{3, j}^{\delta}+\frac{1-2 \kappa_{j m}}{1+\theta_{l, j}^{\delta}-2 \alpha_{j}^{\delta} \theta_{l, j}^{\delta}}\left(r_{4, j}^{\delta}-r_{3, j}^{\delta}\right), & \kappa_{j m} \in\left[\frac{1-\theta_{l, j}^{\delta}+2 \alpha_{j}^{\delta} \theta_{l, j}^{\delta}}{4}, \frac{1}{2}\right] \\ r_{2, j}^{\delta}-\frac{2 \kappa_{j m}-1}{1+\theta_{l, j}^{\delta}-2 \alpha_{j}^{\delta} \theta_{l, j}^{\delta}}\left(r_{2, j}^{\delta}-r_{1, j}^{\delta}\right), & \kappa_{j m} \in\left(\frac{1}{2}, \frac{3+\theta_{l, j}^{\delta}-2 \alpha_{j}^{\delta} \theta_{l, j}^{\delta}}{4}\right] \\ r_{1, j}^{\delta}+\frac{2-2 \kappa_{j m}}{1-\theta_{l, j}^{\delta}+2 \alpha_{j}^{\delta} \theta_{l, j}^{\delta}}\left(r_{2, j}^{\delta}-r_{1, j}^{\delta}\right), & \kappa_{j m} \in\left[\frac{3+\theta_{l, j}^{\delta}-2 \alpha_{j}^{\delta} \theta_{l, j}^{\delta}}{4}, 1\right] .\end{cases}
$$

Proof. It can be proved similarly as Theorem 5 .

4.2. Deterministic Parametric Model. On the basis of Theorems $3,4,5$, and 6, we can transform the original model (22)(33) into its equivalent model as follows:

$$
\begin{array}{ll}
\max & \beta \\
\text { s.t. } & f_{0}-\sum_{j \in J} \sum_{l \in L} g_{j l} w_{j l}-\sum_{i \in I} \sum_{j \in J} \sum_{m \in M} a_{i m} x_{i j m}-\sum_{i \in I} \sum_{j \in J} \sum_{m \in M} b_{i j m} x_{i j m} \\
& \geq r\left(\sum_{i \in I} \sum_{j \in J} \sum_{m \in M} \widetilde{\xi}_{i m} y_{i j m}+\sum_{i \in I} \sum_{j \in J} \sum_{m \in M} e_{i j} \widetilde{\eta}_{m} y_{i j m}+\sum_{j \in J} \sum_{k \in K} \sum_{m \in M} E_{j k} \widetilde{\zeta}_{m} z_{j k m}\right)_{\text {inf }}(\beta), \\
& \sum_{l \in L} w_{j l} \leq 1, \quad \forall j \in J
\end{array}
$$




$$
\begin{aligned}
& \sum_{i \in I} y_{i j m} \leq Q \sum_{l \in L} w_{j l}, \quad \forall j \in J, m \in M \\
& \sum_{k \in K} z_{j k m} \leq Q \sum_{l \in L} w_{j l}, \quad \forall j \in J, m \in M \\
& \sum_{j \in J} x_{i j m} \leq S_{i m}, \quad \forall i \in I, m \in M \\
& \sum_{i \in I} \sum_{m \in M} c_{m} x_{i j m} \leq \sum_{l \in L} v_{l} w_{j l}, \quad \forall j \in J \\
& \sum_{j \in J} z_{j k m} \geq d_{k m, \text { inf }}\left(\phi_{k m}\right), \quad \forall k \in K, m \in M \\
& \sum_{j \in J} y_{i j m} \leq \lambda_{i, \text { sup }}\left(\psi_{i m}\right) p_{m} S_{i m}, \quad \forall i \in I, m \in M \\
& \sum_{k \in K} z_{j k m}-\sum_{j \in J} y_{i j m} \leq \sum_{i \in I} \delta_{j, s u p}\left(\kappa_{j m}\right) p_{m} x_{i j m}, \quad \forall j \in J, m \in M \\
& w_{j l} \in\{0,1\}, \quad \forall j \in J, l \in L \\
& x_{i j m}, y_{i j m}, z_{j k m} \geq 0, \quad \forall i \in I, \quad j \in J, k \in K, m \in M,
\end{aligned}
$$

where $r(\cdot)_{\text {inf }}(\beta), d_{k m \text {,inf }}\left(\phi_{k m}\right), \lambda_{i, \text { sup }}\left(\psi_{i m}\right)$, and $\delta_{j \text {,sup }}\left(\kappa_{j m}\right)$ are given by (39), (48), (53), and (57), respectively.

The equivalent model (58) is parametric nonlinear programming. In addition, there are $0-1$ integer decision variables, so it is a mixed-integer program, which can be solved by branch-and-bound method. The LINGO is a state-of-the-art optimization tool including the branch-and-bound IP code and can be used to solve model (58).

\section{Illustrative Example}

In this section, we conduct a collection of numerical experiments to show the effectiveness of our developed model.

5.1. Test Problem. We now consider an example focusing on preparedness for earthquake threats. There are five affected areas that need to provide emergency responses. In order to give shelter and assistance to disaster victims as soon as possible, an emergency distribution center of any size can be opened at any one of four candidate locations in the network. Without loss of generality, three possible sizes of facilities are considered with fixed parameters as shown in Table 1. After disaster, since many resources are scarce, the related department is in charge of acquiring emergency supplies from suppliers and dispatching them to the disaster areas. The unit acquisition costs and the maximal supply capacity for suppliers are displayed in Table 2 . The emergency resources stored in the distribution center contain two kinds of supplies: food and clothes. The costs and space estimates for acquiring, storing, and allocating these supplies are provided in Table 3. In the given network, the distances among different facilities, suppliers, distribution centers, and affected areas, are collected in Table 4. Because of the uncertainty about whether or not disasters will occur, and if they do, where and with what magnitude, the accurate values of cost, demand, and damage level are not known prior to disaster and characterized by type- 2 trapezoidal fuzzy variables shown in Tables 5 and 6. Our goal is to find an optimal strategy for prepositioning and allocating the emergency supplies such that the total cost is no more than 105,000 .

5.2. Optimal Prepositioning Plan. We next employ LINGO software to solve our prepositioning emergency supplies model. For the sake of presentation, suppose that the parameters $\theta$ 's take their values as shown in Table 7 . We set the parameters $\alpha_{i}^{\lambda}=\alpha_{j}^{\delta}=\alpha_{k m}^{d}=0.35$ for any index $i, j, k, m$, and $\alpha=0.85$. When the threshold values in credibility constraints are set to $\phi_{k m}=\psi_{i m}=\kappa_{j m}=0.95$, we obtain that the corresponding optimal solution is reported in Figure 2 with the credibility more than 0.948 .

From Figure 2, we first know that three distribution centers are opened, located in the candidate locations 1,2 , and 3. Moreover, the second distribution center is located in the medium category, and the first and third ones are located in the small category. Then, Figure 2 also tells us the quantities of various types of emergency supplies prepositioned as well as the amounts of every commodity replenished once an earthquake occurs. The predisaster acquisition amounts of emergency supplies are marked by 2-dimension vector in the thin lines between suppliers and distribution centers, while the postdisaster replenishment amounts of emergency supplies are labelled by 2 -dimension vector in the thick lines. For example, the number pair $(77,168)$ in thin link between 


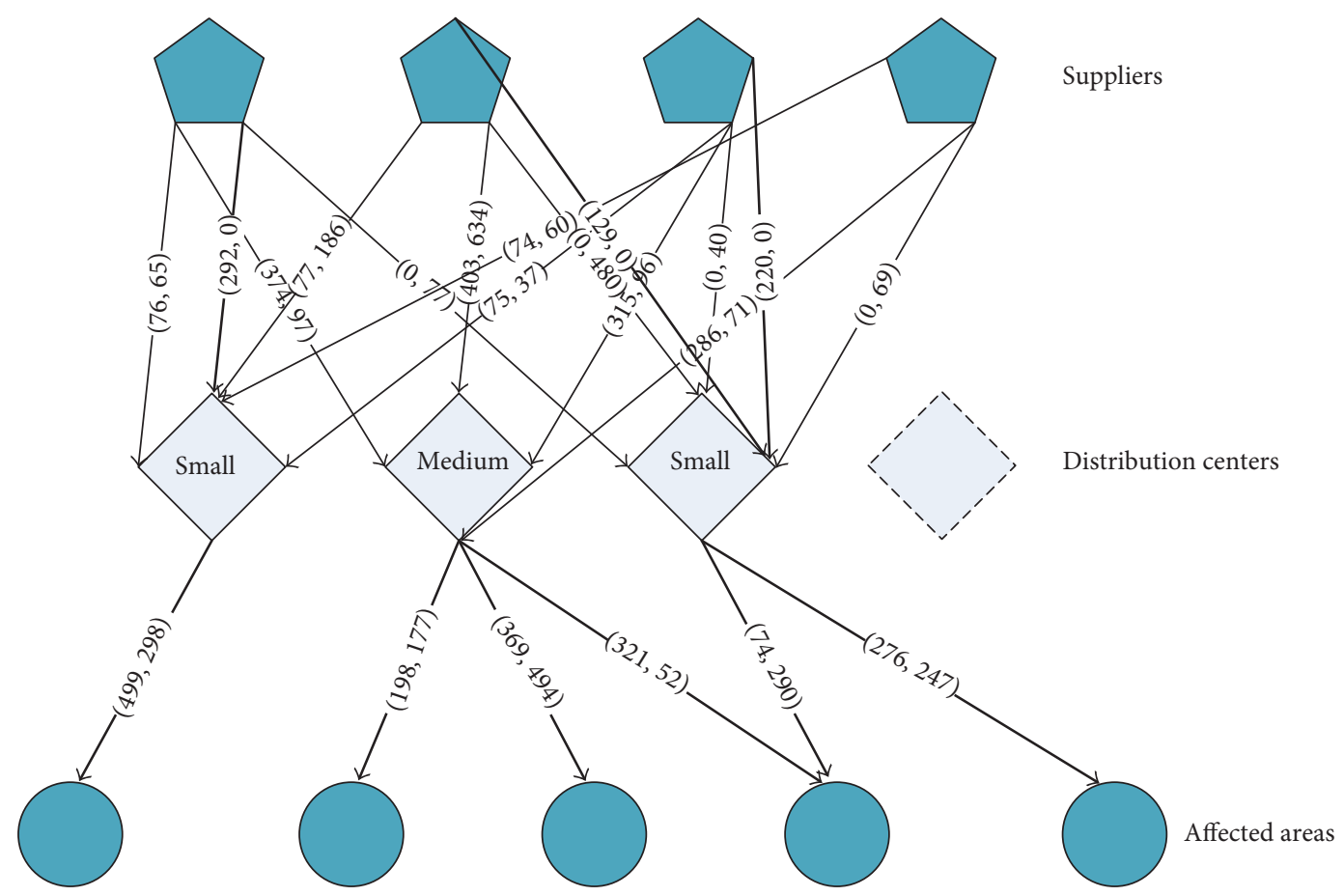

FIGURE 2: Solution result for prepositioning emergency supplies model.

TABLE 1: Parameters related to different distribution centers.

\begin{tabular}{lccc}
\hline $\begin{array}{l}\text { Size category } \\
\text { of } \\
\text { distribution } \\
\text { center }\end{array}$ & Descriptor & $g_{j l}$ & $v_{l}$ \\
\hline 1 & Small & 650 & 1,000 \\
2 & Medium & 1,100 & 5,000 \\
3 & Large & 1,350 & 8,000 \\
\hline
\end{tabular}

TABLE 2: Parameters related to different suppliers.

\begin{tabular}{ccccccccc}
\hline & \multicolumn{3}{c}{$a_{i m}$} \\
$m$ & \multicolumn{3}{c}{$i$} & & \multicolumn{5}{c}{$S_{\text {im }}$} \\
& 1 & 2 & 3 & 4 & 1 & 2 & 3 & 4 \\
\hline 1 & 6.2 & 5.8 & 5.4 & 5.5 & 450 & 480 & 390 & 360 \\
2 & 10 & 13 & 20 & 12 & 240 & 1,300 & 800 & 200 \\
\hline
\end{tabular}

the second supplier and the first distribution center indicates that 77 units of food and 168 units of clothes from the second supplier are prepositioned in the first distribution center before disaster. The number pair $(220,0)$ in thick link between the third supplier and the third distribution center implies that 220 units of food from the third supplier are replenished in the third distribution center after disaster and the other relief is abundant. Not only that, but also we can find that all the replenishment amounts for the second relief are zero. We now give some explanations about the computational results. Food belongs to perishable goods, so it is hard to maintain a high inventory for the distribution center. Usually, before the realized value of fuzzy variable demand is known, its conventional storage is low. Finally, Figure 2 also provides us the allocation mode of various types of emergency supplies. For example, the third distribution center is mainly responsible for the basic demands from the fourth and fifth affected areas. The number pair $(74,290)$ in thick line between the third distribution center and the fourth affected area indicates that 74 units of food and 290 units of clothes are transported from the third distribution center to the fourth affected area after disaster. Meanwhile, the number pair $(276,247)$ in thick line between the third distribution center and the fifth affected area indicates that the third distribution center provides 276 units of food and 247 units of clothes to the fourth affected area.

5.3. Parameter Analysis. In order to identify the impact of model parameter $f_{0}$ in the objective on solution quality, we find optimal solutions by adjusting the values of parameter $f_{0}$, changing it from 100000 to 106000 by 1000 , and report the computational results in Figure 3.

Figure 3 shows that there is much difference in terms of the optimal objective value when the parameter $f_{0}$ changes. Furthermore, if the acceptable threshold turns large, the credibility of the total cost not exceeding $f_{0}$ has the increasing trend. So it is concluded that there exist some positive correlations between the credibility and the threshold $f_{0}$.

5.4. Comparative Study. In order to demonstrate the advantage of using type- 2 fuzzy data, we compare the proposed model to the credibility constrained type-1 fuzzy prepositioning emergency supplies model. 
Table 3: Parameters related to different emergency supplies.

\begin{tabular}{lccccc}
\hline Supply & $b_{m}$ & $c_{m}$ & $p_{m}$ & $\widetilde{\eta}_{m}$ & $\widetilde{\zeta}_{m}$ \\
\hline Food & 0.1 & 1 & 0.8 & $\left(0.12,0.15,0.18,0.2 ; \theta_{l, 1}^{\eta}, \theta_{r, 1}^{\eta}\right)$ & $\left(0.12,0.18,0.2,0.25 ; \theta_{l, 1}^{\zeta}, \theta_{r, 1}^{\zeta}\right)$ \\
Clothes & 0.2 & 1.5 & 1 & $\left(0.2,0.25,0.28,0.4 ; \theta_{l, 2}^{\eta}, \theta_{r, 2}^{\eta}\right)$ & $\left(0.25,0.3,0.35,0.45 ; \theta_{l, 2}^{\zeta}, \theta_{r, 2}^{\zeta}\right)$ \\
\hline
\end{tabular}

TABLE 4: Parameters related to distances of different network nodes.

\begin{tabular}{cccccccccc}
\hline$j$ & & $i$ & & & & $k$ & \\
& 1 & 2 & 3 & 4 & 1 & 2 & 3 & 40 \\
\hline 1 & 30 & 110 & 80 & 90 & 60 & 70 & 90 & 130 \\
2 & 150 & 70 & 50 & 90 & 80 & 50 & 40 & 60 \\
3 & 130 & 60 & 40 & 90 & 100 & 70 & 120 & 50 & 120 \\
4 & 100 & 120 & 50 & 70 & 90 & 50 & 130 & 60 \\
\hline
\end{tabular}

TABLE 5: Parameters related to acquisition costs and proportions of different emergency supplies.

\begin{tabular}{ccc}
\hline$i$ & \multicolumn{3}{c}{$m$} \\
\hline & 1 & 2 \\
\hline 1 & $\left(4.96,6.2,9.3,12.4 ; \theta_{l, 11}^{\xi}, \theta_{l, 11}^{\xi}\right)$ & $\left(8,10,15,20 ; \theta_{l, 12}^{\xi}, \theta_{l, 11}^{\xi}\right)$ \\
2 & $\left(4.64,5.8,8.7,11.6 ; \theta_{l, 21}^{\xi}, \theta_{l, 21}^{\xi}\right)$ & $\left(10.4,13,19.5,26 ; \theta_{l, 22}^{\xi}, \theta_{l, 22}^{\xi}\right)$ \\
3 & $\left(4.32,5.4,8.1,10.8 ; \theta_{l, 31}^{\xi}, \theta_{l, 31}^{\xi}\right)$ & $\left(16,20,30,40 ; \theta_{l, 32}^{\xi}, \theta_{l, 32}^{\xi}\right)$ \\
4 & $\left(4.4,5.5,8.25,11 ; \theta_{l, 41}^{\xi}, \theta_{l, 41}^{\xi}\right)$ & $\left(9.6,12,18,24 ; \theta_{l, 42}^{\xi}, \theta_{l, 42}^{\xi}\right)$ \\
\hline & $i$ & $j$ \\
\hline 1 & $\left(0.8,0.9,0.96,1 ; \theta_{l, 1}^{\lambda}, \theta_{l, 1}^{\lambda}\right)$ & $\left(0.85,0.9,0.91,0.97 ; \theta_{l, 1}^{\delta}, \theta_{l, 1}^{\delta}\right)$ \\
2 & $\left(0.5,0.6,0.7,0.75 ; \theta_{l, 2}^{\lambda}, \theta_{l, 2}^{\lambda}\right)$ & $\left(0.8,0.84,0.88,1 ; \theta_{l, 2}^{\delta}, \theta_{l, 2}^{\delta}\right)$ \\
3 & $\left(0.7,0.75,0.8,0.9 ; \theta_{l, 3}^{\lambda}, \theta_{l, 3}^{\lambda}\right)$ & $\left(0.8,0.85,0.9,0.95 ; \theta_{l, 3}^{\delta}, \theta_{l, 3}^{\delta}\right)$ \\
4 & $\left(0.8,0.82,0.9,0.95 ; \theta_{l, 4}^{\lambda}, \theta_{l, 4}^{\lambda}\right)$ & $\left(0.8,0.85,0.9,1 ; \theta_{l, 4}^{\delta}, \theta_{l, 4}^{\delta}\right)$ \\
\hline
\end{tabular}

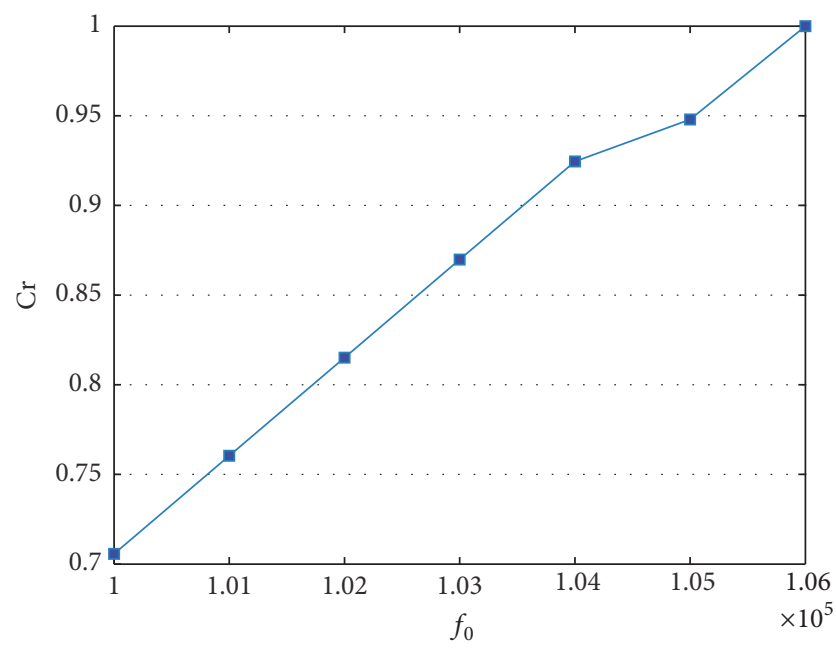

Figure 3: Parameter analysis about $f_{0}$.

In a type- 2 trapezoidal fuzzy variable $\widetilde{\xi}=\left(\widetilde{r}_{1}, \widetilde{r}_{2}, \widetilde{r}_{3}\right.$, $\left.\tilde{r}_{4} ; \theta_{l}, \theta_{r}\right)$, the parameters $\theta_{l}$ and $\theta_{r}$ are used to describe the degrees of uncertainty. When $\theta_{l}=\theta_{r}=0$, the type- 2 fuzzy variable degenerates to its corresponding type-1 trapezoidal fuzzy variable $\bar{\xi}=\left(r_{1}, r_{2}, r_{3}, r_{4}\right)$. In this way, it is easy to obtain the related type-1 trapezoidal fuzzy data $\bar{\xi}_{i m}, \bar{\eta}_{m}, \bar{\zeta}_{m}, \bar{d}_{k m}, \bar{\lambda}_{i}$,
TABLE 6: Parameters related to demands of different affected areas.

\begin{tabular}{ccc}
\hline$k$ & \multicolumn{3}{c}{$m$} \\
\hline 1 & $\left(455,460,490,500 ; \theta_{l, 11}^{d}, \theta_{l, 11}^{d}\right)$ & $\left(240,250,280,300 ; \theta_{l, 12}^{d}, \theta_{l, 11}^{d}\right)$ \\
2 & $\left(120,160,180,200 ; \theta_{l, 21}^{d}, \theta_{l, 21}^{d}\right)$ & $\left(80,120,160,180 ; \theta_{l, 22}^{d}, \theta_{l, 22}^{d}\right)$ \\
3 & $\left(280,330,360,370 ; \theta_{l, 31}^{d}, \theta_{l, 31}^{d}\right)$ & $\left(300,400,450,500 ; \theta_{l, 32}^{d}, \theta_{l, 32}^{d}\right)$ \\
4 & $\left(280,315,360,400 ; \theta_{l, 41}^{d}, \theta_{l, 41}^{d}\right)$ & $\left(200,260,290,350 ; \theta_{l, 42}^{d}, \theta_{l, 42}^{d}\right)$ \\
5 & $\left(200,235,250,280 ; \theta_{l, 51}^{d}, \theta_{l, 51}^{d}\right)$ & $\left(160,220,230,250 ; \theta_{l, 52}^{d}, \theta_{l, 52}^{d}\right)$ \\
\hline
\end{tabular}

and $\bar{\delta}_{j}$ in Tables 3,5 , and 6 . By making use of the type-1 fuzzy numbers, we formulate model (59) as follows:

$$
\begin{array}{ll}
\max & \beta \\
\text { s.t. } & \operatorname{Cr}\left\{f(\mathbf{w}, \mathbf{x}, \mathbf{y}, \mathbf{z} ; \overline{\boldsymbol{\xi}}, \overline{\boldsymbol{\eta}}, \overline{\boldsymbol{\zeta}}) \leq f_{0}\right\} \geq \beta \\
& \sum_{l \in L} w_{j l} \leq 1, \quad \forall j \in J \\
& \sum_{i \in I} y_{i j m} \leq Q \sum_{l \in L} w_{j l}, \quad \forall j \in J, m \in M \\
& \sum_{k \in K} z_{j k m} \leq Q \sum_{l \in L} w_{j l}, \quad \forall j \in J, m \in M \\
& \sum_{j \in J} x_{i j m} \leq S_{i m}, \quad \forall i \in I, m \in M \\
& \sum_{i \in I} \sum_{m \in M} c_{m} x_{i j m} \leq \sum_{l \in L} v_{l} w_{j l}, \quad \forall j \in J \\
& \operatorname{Cr}\left\{\sum_{j \in J} z_{j k m} \geq \bar{d}_{k m}\right\} \geq \phi_{k m},
\end{array}
$$

$\forall k \in K, m \in M$$$
\operatorname{Cr}\left\{\sum_{j \in J} y_{i j m} \leq \bar{\lambda}_{i m} S_{i m}\right\} \geq \psi_{i m}
$$

$\forall i \in I, m \in M$ 
TABLE 7: Values of parameter $\theta$.

\begin{tabular}{lccccccccc}
\hline$\theta$ & 1 & 2 & 3 & 4 & 5 & 6 & 7 & 8 & 9 \\
\hline$\theta_{l, m}^{\eta}$ & 0.68 & 0.49 & & & & & & \\
$\theta_{r, m}^{\eta}$ & 0.74 & 0.36 & & & & & & \\
$\theta_{l, m}^{\zeta}$ & 0.91 & 0.65 & & & & & & \\
$\theta_{r, m}^{\zeta}$ & 0.62 & 0.79 & & & & & & \\
$\theta_{l, i}^{\lambda}$ & 0.23 & 0.18 & 0.76 & 0.85 & & & & \\
$\theta_{r, i}^{\lambda}$ & 0.34 & 0.28 & 0.95 & 0.78 & & & & \\
$\theta_{l, j}^{\delta}$ & 0.54 & 0.83 & 0.26 & 0.69 & & & & \\
$\theta_{r, j}^{\delta}$ & 0.48 & 0.86 & 0.94 & 0.52 & & & & \\
$\theta_{l, i m}^{\xi}$ & 0.97 & 0.45 & 0.87 & 0.26 & 0.73 & 0.63 & 0.59 & & \\
$\theta_{r, i m}^{\xi}$ & 0.26 & 0.62 & 0.92 & 0.68 & 0.94 & 0.46 & 0.72 & 0.93 & \\
$\theta_{l, k m}^{d}$ & 0.47 & 0.28 & 0.35 & 0.85 & 0.96 & 0.58 & 0.61 & 0.93 & 0.58 \\
$\theta_{r, k m}^{d}$ & 0.18 & 0.67 & 0.48 & 0.93 & 0.54 & 0.89 & 0.55 & 0.73 & 0.36 \\
\hline
\end{tabular}

$$
\begin{array}{r}
\operatorname{Cr}\left\{\sum_{i \in I}\left(\bar{\delta}_{j m} x_{i j m}+y_{i j m}\right) \geq \sum_{k \in K} z_{j k m}\right\} \geq \kappa_{j m}, \\
\forall j \in J, m \in M \\
w_{j l} \in\{0,1\}, x_{i j m}, y_{i j m}, z_{j k m} \geq 0 \\
\forall i \in I, j \in J, k \in K, l \in L, m \in M .
\end{array}
$$

The uncertain parameters in model (22)-(33) are CVaR reduced fuzzy variables which are not always normalized, while those data in model (59) are normalized type-1 trapezoidal fuzzy variables. So models (59) and (22)-(33) are different.

For the type-1 numerical example, we use Theorems 3, 4, 5 , and 6 to develop the equivalent transformation of model (59) in case of $\theta_{l}=\theta_{r}=0$, denoted by model (60), which can correspond to the prepositioning problem in the type-1 fuzzy environment. For $0.5<\phi_{k m}, \psi_{i m}, \kappa_{j m} \leq 1$,

$$
\begin{array}{ll}
\max & \beta \\
\text { s.t. } \quad & \sum_{j \in J} \sum_{l \in L} g_{j l} w_{j l}+\sum_{i \in I} \sum_{j \in J} \sum_{m \in M} a_{i m} x_{i j m}+\sum_{i \in I} \sum_{j \in J} \sum_{m \in M} b_{i j m} x_{i j m} \\
& +\sum_{i \in I} \sum_{j \in J} \sum_{m \in M}\left(r_{4, i m}^{\xi}-2(1-\beta)\left(r_{4, i m}^{\xi}-r_{3, i m}^{\xi}\right)\right) y_{i j m} \\
& +\sum_{i \in I} \sum_{j \in J} \sum_{m \in M} e_{i j}\left(r_{4, m}^{\eta}-2(1-\beta)\left(r_{4, m}^{\eta}-r_{3, m}^{\eta}\right)\right) y_{i j m} \\
& +\sum_{j \in J} \sum_{k \in K} \sum_{m \in M} E_{j k}\left(r_{4, m}^{\zeta}-2(1-\beta)\left(r_{4, m}^{\zeta}-r_{3, m}^{\zeta}\right)\right) z_{j k m} \\
\leq & f_{0}, \quad \forall j \in J \\
& \sum_{l \in L} w_{j l} \leq 1, \quad \forall j \in M \\
& \sum_{i \in I} y_{i j m} \leq Q \sum_{l \in L} w_{j l}, \quad \forall j \in J, m \in M \\
& \sum_{k \in K} z_{j k m} \leq Q \sum_{l \in L} w_{j l}, \quad \forall j \in J, \quad m \in M
\end{array}
$$

$$
\begin{aligned}
& \sum_{j \in J} x_{i j m} \leq S_{i m}, \quad \forall i \in I, m \in M \\
& \sum_{i \in I} \sum_{m \in M} c_{m} x_{i j m} \leq \sum_{l \in L} v_{l} w_{j l}, \quad \forall j \in J \\
& \sum_{j \in J} z_{j k m} \geq r_{4, k m}^{d}-2\left(1-\phi_{k m}\right)\left(r_{4, k m}^{d}-r_{3, k m}^{d}\right),
\end{aligned}
$$

$\forall k \in K, m \in M$

$$
\sum_{j \in J} y_{i j m} \leq\left(r_{1, i}^{\lambda}+2\left(1-\psi_{i m}\right)\left(r_{2, i}^{\lambda}-r_{1, i}^{\lambda}\right)\right) p_{m} S_{i m}
$$

$\forall i \in I, m \in M$

$$
\begin{aligned}
& \sum_{k \in K} z_{j k m}-\sum_{j \in J} y_{i j m} \\
& \leq \sum_{i \in I}\left(r_{1, j}^{\delta}+2\left(1-\kappa_{j m}\right)\left(r_{2, j}^{\delta}-r_{1, j}^{\delta}\right)\right) p_{m} x_{i j m}, \\
& \forall j \in J, m \in M \\
& w_{j l} \in\{0,1\}, x_{i j m}, y_{i j m}, z_{j k m} \geq 0, \quad \\
& \forall i \in I, \quad j \in J, k \in K, l \in L, m \in M .
\end{aligned}
$$

When we set the threshold values $\phi_{k m}=\psi_{i m}=\kappa_{j m}=0.95 \mathrm{in}$ credibility constraints, the corresponding optimal credibility is more than 0.9804 .

From the above statement, it is clear that the result solved by the type-1 fuzzy prepositioning emergency supplies model is only a special case of the type- 2 fuzzy model. The reason is stated as follows. On the one hand, in the type-1 fuzzy model, the uncertain data are assumed to have fixed possibility distribution, which usually depend on the decision maker's knowledge and experience. Under this environment, the optimal prepositioning strategy is related to the fixed possibility distributions. If the fixed possibility distribution cannot be determined accurately, then it is unmeaningful that we adopt such a solution. On the other hand, in our type- 2 fuzzy prepositioning model, the uncertain parameters are described by variable possibility distribution. Once it is difficult to determine the fixed possibility distributions, the proposed parametric optimization method paves an 
effective way for decision maker. So, the decision makers can identify the informed emergency supplies schedule. In brief, the comparative study implies that the variable possibility distributions have some advantages over the fixed possibility distributions, such as alleviating the burden of the decision maker, avoiding the loss of information, and guaranteeing the accuracy of the prepositioning results.

\section{Conclusions}

In this paper, we studied a new fuzzy prepositioning emergency supplies problem, including model formulation, property analysis, and numerical experiments. We next summarized the main results of the paper.

(i) Theorems 1 and 2 present the optimistic value and pessimistic value formula for the CVaR reduced fuzzy variables of type-2 trapezoidal fuzzy variable. Using the formulas, we can lessen the complexity of computing the credibility constraints so that much time can be saved when solving the proposed prepositioning model.

(ii) We established a new fuzzy maximal credibility prepositioning emergency supplies model, in which the postdisaster acquisition and transportation costs, the damage level, and affected areas' demand were uncertain and assumed to be type- 2 fuzzy variables.

(iii) On the basis of the CVaR reduction method and obtained formula, Theorem 3 discussed the equivalent expression of chance objective, while Theorems 4-6 gave the parametric forms of credibility constraints. Thus, we converted the original optimization problem into its equivalent parametric programming model and solved the corresponding optimal solutions through LINGO software.

(iv) We provided a numerical example to demonstrate the effectiveness of the proposed model. The computational results showed that the variable possibility distributions have some advantages over the fixed possibility distributions for prepositioning problem.

There are several directions for our future research. On the one hand, we did not consider the availability of path after disaster. Once some links were unusable, the transportation of emergency supplies would use more circuitous routes. The case of adding other transportation modes such as air and sea transportation is an important issue for further research. On the other hand, we assumed the possibility distributions of the type-2 fuzzy variables were trapezoidal. If not, it is unsuitable for our proposed model to employ the general-purpose software. Under this condition, it is of special value to develop a more efficient customized evolutionary algorithm [32, 33], like GA, PSO, and BBO, by exploiting the structure of the prepositioning model.

\section{Notations}

Indices

I: Set of suppliers, indexed by $i$

$J$ : Set of distribution centers, indexed by $j$

$K$ : Set of affected areas, indexed by $k$

$L$ : Set of size categories for each distribution center, indexed by $l$

$M$ : Set of emergency supplies, indexed by $m$.

\section{Fixed Parameters}

$a_{i m}$ : Unit acquisition cost for emergency relief $m$ obtained from supplier $i$

$b_{i j m}$ : Unit transportation cost for emergency relief $m$ from supplier $i$ to distribution center $j$

$c_{m}$ : Unit space volume for emergency relief $m$

$g_{j l}$ : Fixed cost of opening a distribution center $j$ with size category $l$

$v_{l}$ : Maximal allocation capacity of distribution center with size category $l$

$S_{i m}$ : Maximal supply capacity of emergency relief $m$ from supplier $i$

Q: A big real number.

\section{Fuzzy Parameters}

$\widetilde{\xi}_{i m}:$ Type-2 fuzzy acquisition cost for emergency relief $m$ obtained from supplier $i$ after disaster

$\tilde{d}_{k m}$ : Type-2 fuzzy demand for emergency relief $m$ at affected area $k$

$\tilde{\eta}_{i j m}$ : Type-2 fuzzy transportation cost for emergency relief $m$ purchased by supplier $i$ and transported to distribution center $j$

$\widetilde{\zeta}_{j k m}$ : Type-2 fuzzy transportation cost for emergency relief $m$ shipped from distribution center $j$ to affected area $k$

$\tilde{\lambda}_{i m}:$ Type-2 fuzzy proportion of stocked material for emergency relief $m$ at supplier $i$ after disaster

$\widetilde{\delta}_{j m}$ : Type-2 fuzzy proportion of stocked material for emergency relief $m$ at distribution center $j$ after disaster.

\section{Decision Variables}

$w_{j l}$ : Binary variable indicating whether a distribution center with size category $l$ is located at node $j$ or not

$x_{i j m}$ : Amount of emergency relief $m$ purchased by supplier $i$ and prepositioned at distribution center $j$

$y_{i j m}$ : Amount of emergency relief $m$ purchased by supplier $i$ and transported to distribution center $j$ after disaster 
$z_{j k m}$ : Amount of emergency relief $m$ transported from distribution center $j$ to affected area $k$.

\section{Competing Interests}

The author declares that there are no competing interests.

\section{Acknowledgments}

This work is supported partially by the National Natural Science Foundation of China (no. 61374184) and the Youth Natural Science Foundation of Hebei Province (no. A2016204057).

\section{References}

[1] R. Abounacer, M. Rekik, and J. Renaud, "An exact solution approach for multi-objective location-transportation problem for disaster response," Computers \& Operations Research, vol. 41, no. 1, pp. 83-93, 2014.

[2] F. Barzinpour and V. Esmaeili, "A multi-objective relief chain location distribution model for urban disaster management," International Journal of Advanced Manufacturing Technology, vol. 70, no. 5-8, pp. 1291-1302, 2014.

[3] N. Altay and W. G. Green III, "OR/MS research in disaster operations management," European Journal of Operational Research, vol. 175, no. 1, pp. 475-493, 2006.

[4] G. Galindo and R. Batta, "Review of recent developments in OR/MS research in disaster operations management," European Journal of Operational Research, vol. 230, no. 2, pp. 201-211, 2013.

[5] A. M. Anaya-Arenas, J. Renaud, and A. Ruiz, "Relief distribution networks: a systematic review," Annals of Operations Research, vol. 223, pp. 53-79, 2014.

[6] L. Özdamar and M. A. Ertem, "Models, solutions and enabling technologies in humanitarian logistics," European Journal of Operational Research, vol. 244, no. 1, pp. 55-65, 2015.

[7] A. Bozorgi-Amiri, M. S. Jabalameli, and S. M. J. M. Al-eHashem, "A multi-objective robust stochastic programming model for disaster relief logistics under uncertainty," OR Spectrum, vol. 35, no. 4, pp. 905-933, 2013.

[8] J. Salmerón and A. Apte, "Stochastic optimization for natural disaster asset prepositioning," Production and Operations Management, vol. 19, no. 5, pp. 561-574, 2010.

[9] C. G. Rawls and M. A. Turnquist, "Pre-positioning of emergency supplies for disaster response," Transportation Research Part B: Methodological, vol. 44, no. 4, pp. 521-534, 2010.

[10] B. Balcik and D. Ak, "Supplier selection for framework agreements in humanitarian relief," Production and Operations Management, vol. 23, no. 6, pp. 1028-1041, 2014.

[11] Z.-H. Zhang and H. Jiang, "A robust counterpart approach to the bi-objective emergency medical service design problem," Applied Mathematical Modelling, vol. 38, no. 3, pp. 1033-1040, 2014.

[12] A. M. Caunhye, Y. Zhang, M. Li, and X. Nie, "A location-routing model for prepositioning and distributing emergency supplies," Transportation Research Part E: Logistics and Transportation Review, vol. 90, pp. 161-176, 2016.

[13] M. C. Hoyos, R. S. Morales, and R. Akhavan-Tabatabaei, "OR models with stochastic components in disaster operations management: a literature survey," Computers \& Industrial Engineering, vol. 82, pp. 183-197, 2015.

[14] J.-B. Sheu, "An emergency logistics distribution approach for quick response to urgent relief demand in disasters," Transportation Research Part E: Logistics and Transportation Review, vol. 43, no. 6, pp. 687-709, 2007.

[15] B. Sun, W. Ma, and H. Zhao, "A fuzzy rough set approach to emergency material demand prediction over two universes," Applied Mathematical Modelling, vol. 37, no. 10-11, pp. 70627070, 2013.

[16] Y.-J. Zheng and H.-F. Ling, "Emergency transportation planning in disaster relief supply chain management: a cooperative fuzzy optimization approach," Soft Computing, vol. 17, no. 7, pp. 1301-1314, 2013.

[17] J. Ruan, P. Shi, C.-C. Lim, and X. Wang, "Relief supplies allocation and optimization by interval and fuzzy number approaches," Information Sciences, vol. 303, pp. 15-32, 2015.

[18] X. Bai, "Two-stage multiobjective optimization for emergency supplies allocation problem under integrated uncertainty," Mathematical Problems in Engineering, vol. 2016, Article ID 2823835, 13 pages, 2016.

[19] S. Tofighi, S. A. Torabi, and S. A. Mansouri, "Humanitarian logistics network design under mixed uncertainty," European Journal of Operational Research, vol. 250, no. 1, pp. 239-250, 2016.

[20] Z.-Q. Liu and Y.-K. Liu, "Type-2 fuzzy variables and their arithmetic," Soft Computing, vol. 14, no. 7, pp. 729-747, 2010.

[21] L. A. Zadeh, "The concept of a linguistic variable and its application to approximate reasoning. I," Information Sciences, vol. 8, pp. 199-249, 1975.

[22] R. Qin, Y. K. Liu, and Z.-Q. Liu, "Modeling fuzzy data envelopment analysis by parametric programming method," Expert Systems with Applications, vol. 38, no. 7, pp. 8648-8663, 2011.

[23] R. Qin, Y.-K. Liu, and Z.-Q. Liu, "Methods of critical value reduction for type-2 fuzzy variables and their applications," Journal of Computational and Applied Mathematics, vol. 235, no. 5, pp. 1454-1481, 2011.

[24] X.-L. Wu and Y.-K. Liu, "Optimizing fuzzy portfolio selection problems by parametric quadratic programming," Fuzzy Optimization and Decision Making, vol. 11, no. 4, pp. 411-449, 2012.

[25] X. Bai and Y. K. Liu, "Semideviations of reduced fuzzy variables: a possibility approach," Fuzzy Optimization and Decision Making, vol. 13, no. 2, pp. 173-196, 2014.

[26] X. J. Bai and Y. K. Liu, "CVAR reduced fuzzy variables and their second order moments," Iranian Journal of Fuzzy Systems, vol. 12, no. 5, pp. 45-75, 2015.

[27] X. Bai and Y. K. Liu, "Robust optimization of supply chain network design in fuzzy decision system," Journal of Intelligent Manufacturing, 2014.

[28] Y. Liu and Y.-K. Liu, "The lambda selections of parametric interval-valued fuzzy variables and their numerical characteristics," Fuzzy Optimization and Decision Making, vol. 15, no. 3, pp. 255-279, 2016.

[29] X.-J. Bai, "Optimization for pre-positioning emergency supplies problem under fuzzy environment," System Engineering Theory and Practice, vol. 35, no. 6, pp. 1465-1473, 2015.

[30] C. G. Rawls and M. A. Turnquist, "Pre-positioning planning for emergency response with service quality constraints," $O R$ Spectrum, vol. 33, no. 3, pp. 481-498, 2011.

[31] Y. K. Liu and X. Bai, "Linear combinations of T2 fuzzy variables," Journal of Uncertain Systems, vol. 8, no. 1, pp. 78-80, 2014. 
[32] L. Wang, J. Song, and L. Shi, "Dynamic emergency logistics planning: models and heuristic algorithm," Optimization Letters, vol. 9, no. 8, pp. 1533-1552, 2015.

[33] Y.-J. Zheng, S.-Y. Chen, and H.-F. Ling, "Evolutionary optimization for disaster relief operations: A survey," Applied Soft Computing Journal, vol. 27, pp. 553-566, 2015. 


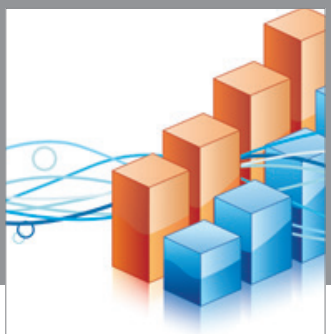

Advances in

Operations Research

vatem alat4

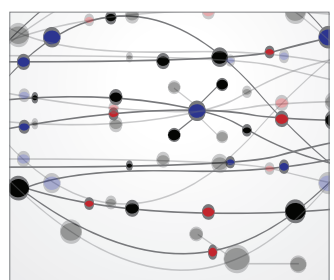

\section{The Scientific} World Journal
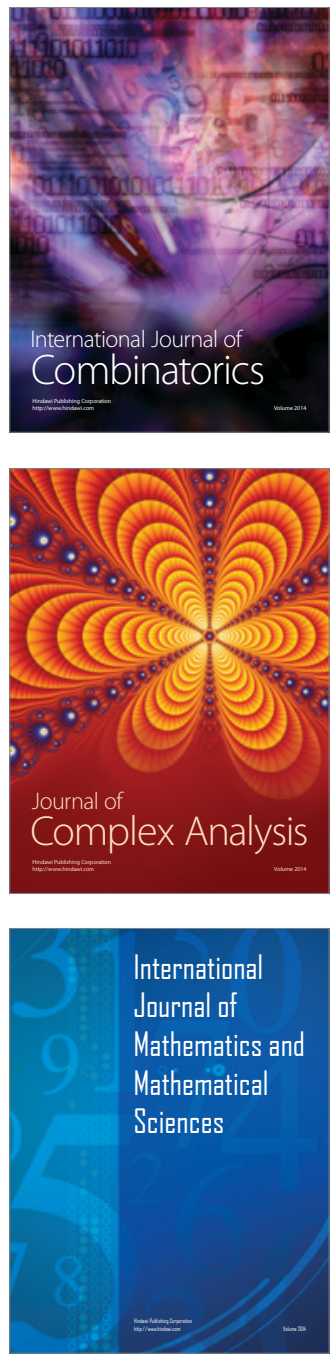
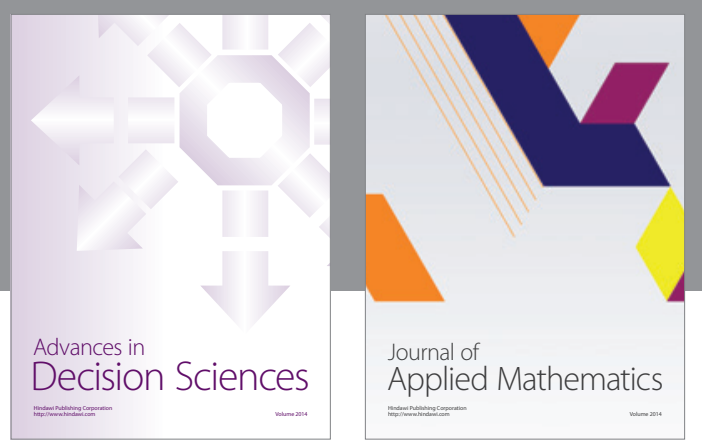

Algebra

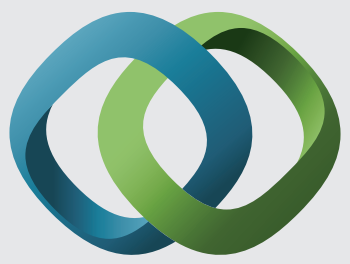

\section{Hindawi}

Submit your manuscripts at

http://www.hindawi.com
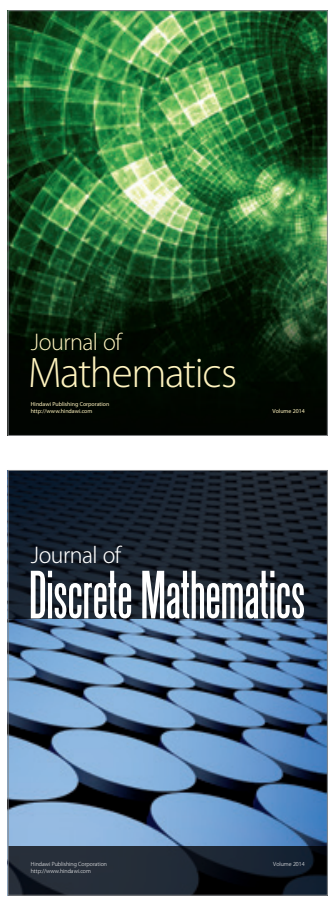

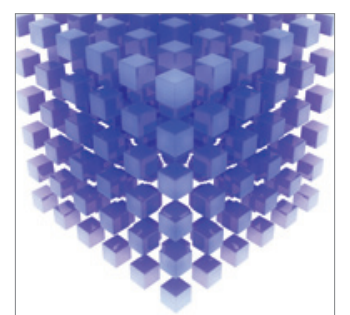

Mathematical Problems in Engineering
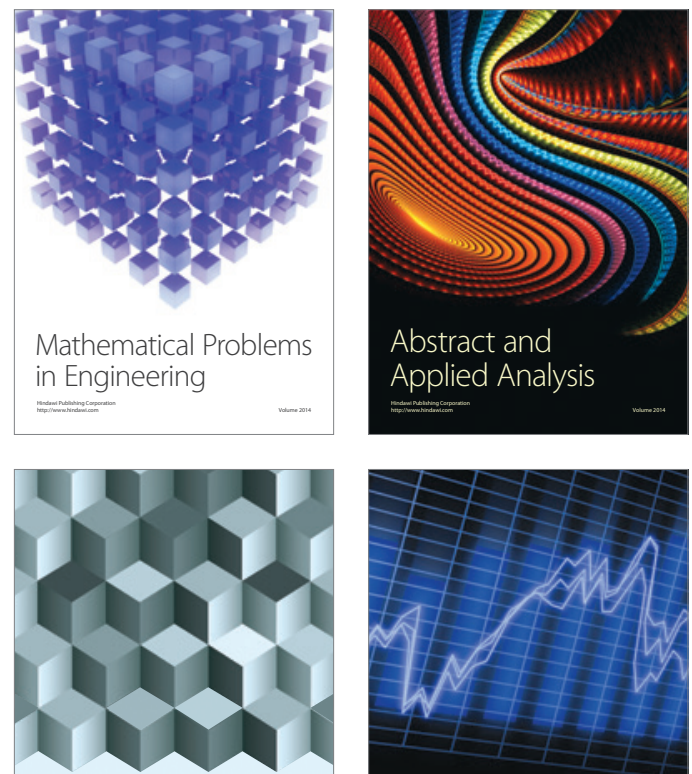

Journal of

Function Spaces

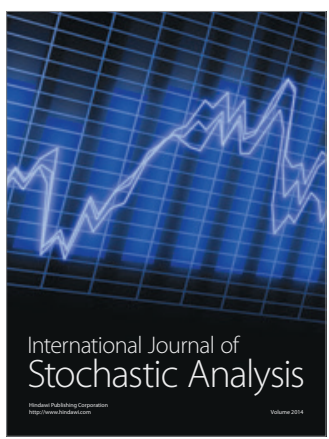

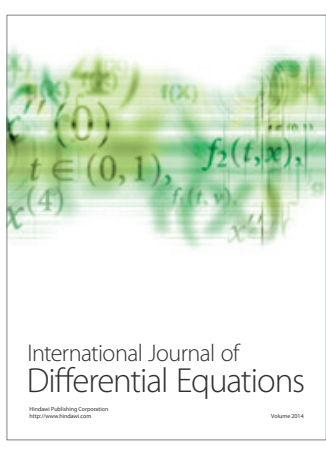
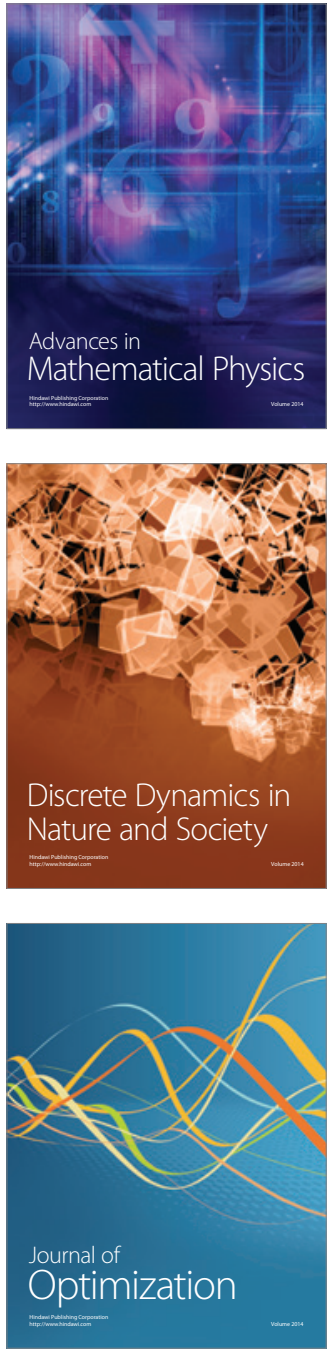